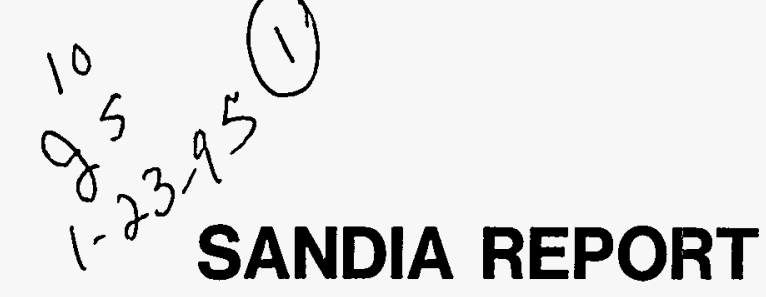

SAND94-2761 $\cdot$ UC-910

Unlimited Release

Printed November 1994

\title{
Optical Generation of Radio-Frequency Power
}

Vincent M. Hietala, G. Allen Vawter, T. M. Brennan, B. E. Hammons, W. Jeffrey Meyer

\section{Prepared by}

Sandla Natlonal Laboratories

Albuquerque, New Mexico 87185 and Livermore, Callfornia 94550

for the Unlted States Department of Energy

under Contract DE-AC04-94ALB 55000

Approved for public release; distribution is unlimited.
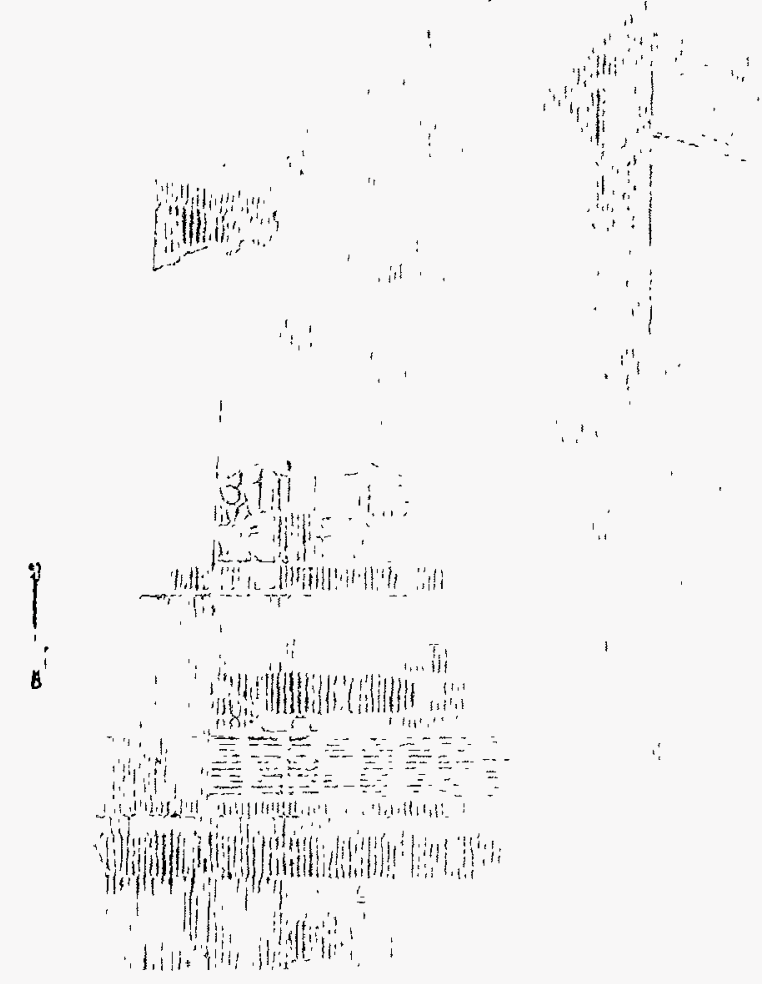
Issued by Sandia National Laboratories, operated for the United States Department of Energy by Sandia Corporation.

NOTICE: This report was prepared as an account of work sponsored by an agency of the United States Government. Neither the United States Government nor any agency thereof, nor any of their employees, nor any of their contractors, subcontractors, or their employees, makes any warranty, express or implied, or assumes any legal liability or responsibility for the accuracy, completeness, or usefulness of any information, apparatus, product, or process disclosed, or represents that its use would not infringe privately owned rights. Reference herein to any specific commercial product, process, or service by trade name, trademark, manufacturer, or otherwise, does not necessarily constitute or imply its endorsement, recommendation, or favoring by the United States Government, any agency thereof or any of their contractors or subcontractors. The views and opinions expressed herein do not necessarily state or reflect those of the United States Government, any agency thereof or any of their contractors.

Printed in the United States of America. This report has been reproduced directly from the best available copy.

Available to DOE and DOE contractors from

Office of Scientific and Technical Information

PO Box 62

Oak Ridge, TN 37831

Prices available from (615) 576-8401, FTS 626-8401

Available to the public from

National Technical Information Service

US Department of Commerce

5285 Port Royal Rd

Springfield, VA 22161

NTIS price codes

Printed copy: A03

Microfiche copy: A01 


\section{DISCLAIMER}

\section{Portions of this document may be illegible in electronic image products. Images are produced from the best available original document.}




\title{
OPTICAL GENERATION OF RADIO-FREQUENCY POWER
}

\author{
Vincent M. Hietala and G. Allen Vawter \\ Compound Semiconductor Technology Department \\ T. M. Brennan, B. E. Hammons \\ Semiconductor Materials Department \\ W. Jeffrey Meyer \\ Advanced Devices \& Applications Department \\ Sandia National Laboratories \\ Albuquerque, New Mexico 87185-0603
}

\begin{abstract}
An optical technique for high-power radio-frequency (RF) signal generation is described. The technique uses a unique photodetector based on a traveling-wave design driven by an appropriately modulated light source. The traveling-wave photodetector (TWPD) exhibits simultaneously a theoretical quantum efficiency approaching $100 \%$ and a very large electrical bandwidth. Additionally, it is capable of dissipating the high-power levels required for the RF generation technique. The modulated light source is formed by either the beating together of two lasers or by the direct modulation of a light source. A system example is given which predicts RF power levels of 100's of mW's at millimeter wave frequencies with a theoretical "wall-plug" efficiency approaching $34 \%$.
\end{abstract}




\section{Acknowledgment}

The authors would like to thank Beth Fuchs for her work in device fabrication, and Mark Housel for his extensive work in device characterization. 


\section{Contents}

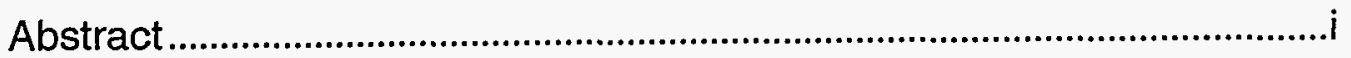

Acknowledgment ...................................................................................i

Contents ..................................................................................................iii

Figures ........................................................................................................

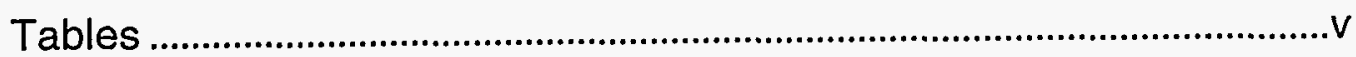

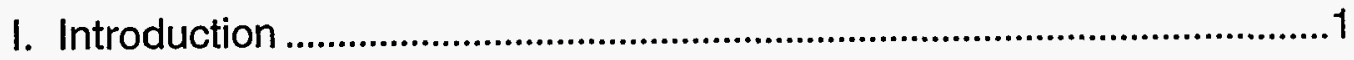

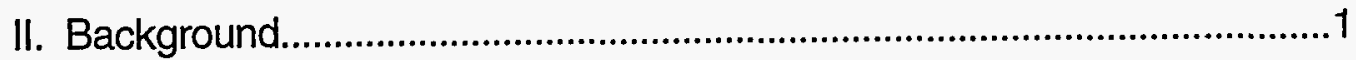

III. Theory of Operation .............................................................................

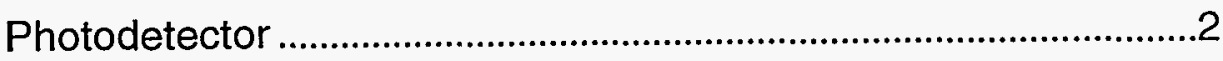

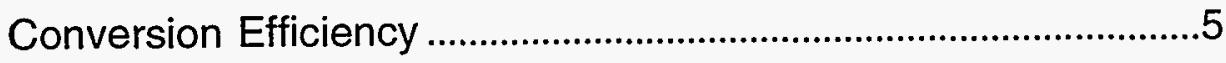

Modulated Light Source ......................................................................

Dual Light Source .....................................................................

Single Light Source ..................................................................11

Component Requirements................................................................11

IV. Traveling-wave Photodetector...............................................................12

Theory of Operation ......................................................................13

Electrode Design ..............................................................................20

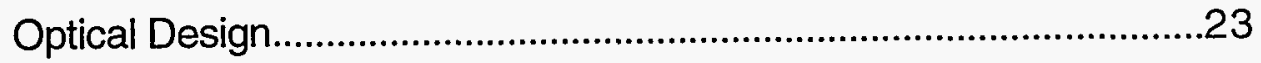

Detector Fabrication ............................................................................27

Detector Characterization ....................................................................29

Electrical Characterization.......................................................30

Optical Bandwidth Measurements ......................................32

V. System Example....................................................................................33

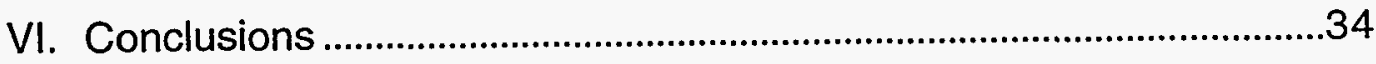


References.

Appendix, LDRD Summary.

\section{Figures}

1. State-of-the-art of solid-state RF power generation...............................

2. RF output power verses peak optical power. .......................................... 4

3. Ideal modulated light source for RF generation. .................................. 7

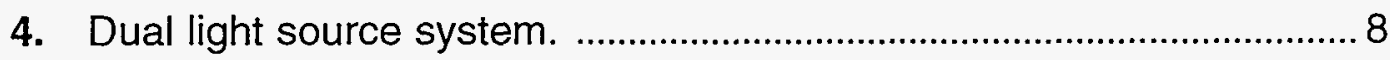

5. Wavelength control of dual light source system. .................................10

6. Injection locking of dual light source system. .......................................10

7. Single light source system. ..................................................................11

8. Diagram of traveling-wave photodetector. ..........................................12

9. Equivalent transmission-line circuit for TWPD. ……..........................13

10. Theoretical TWPD response with output terminated in $Z_{O}$ calculated for several different optical adsorption values.

11. Theoretical TWPD response with both input and output terminated in $\mathrm{Z}_{0}$...

12. Theoretical TWPD response with $\alpha_{0}=1 \mathrm{~mm}^{-1}$ for both input load $\mathrm{P}_{\mathrm{RI}}$ and output load $\mathrm{P}_{\mathrm{RO}}$.

13. Theoretical TWPD response as function of RF index and frequency.

14. Dimensioned simplified cross-section of TWPD for electrical propagation analysis.

15. Theoretical microwave RF Index and RF Loss. ...................................22

16. Theoretical microwave characteristic impedance. ...............................23

17. Cross-section schematic of the TWPD for $1.06 \mu \mathrm{m}$ operation (top) and diagram of material composition and thicknesses through the center axis of the waveguide (bottom). 
18. Contour plot of constant electric field of the TEO0 mode of our TWPD.

19. Top view of Traveling-wave photodetector. …….................................26

20. Theoretical detector output power for the device parameters given in Tables 1-3.

21. SEM micrograph of top view of traveling-wave photodetector. 28

22. SEM micrograph of electrical pad interface to center conductor of the optical guide.

23. SEM micrograph of "air-bridge" connection to center electrode on top of the optical guide.

24. Measured RF index of the TWPD. 30

25. Measured characteristic impedance of the TWPD. 31

26. Measured attenuation of the TWPD. 31

27. Response measurement of the TWPD 33

28. Example: Dual Laser System. 34

\section{Tables}

1. Parameters used for TWPD calculations. 16

2. Parameters used for TWPD electrical propagation calculations. .....22

3. Exciton resonance of quantum well in terms of $\mu \mathrm{m}$ wavelength. ......25

4. Parameters used for TWPD performance calculations. 


\section{Introduction}

Present solid-state technologies for millimeter-wave $(>30 \mathrm{GHz})$ signal generation exhibit low power and narrowband operation, and generally poor reliability. Described here is the approach of using a simple optical generation technique which promises to produce 100's of mW's of power at frequencies from audio to millimeter waves. The system is based on laser light sources, which have demonstrated device lifetimes of thousands of hours, and a unique traveling-wave photodetector of which has no known failure mode. Hence, the technique should exhibit a high degree of reliability. The broadband nature of the technique would allow for an additional cost savings as a standard generation module which could be used for numerous applications at various operating frequencies.

The basis for our technique is that optical detectors produce a current which is proportional to the incident optical power and the electrical power available from a current source is theoretically infinite. Therefore, if appropriate optical detectors can be constructed and appropriate impedance matching realized, significant RF power levels should be possible from an optical system. This report summarizes the results of a Laboratory Directed Research and Development (LDRD) project performed at Sandia National Laboratories (case \#3512.040).

\section{Background}

Fig. 1 shows the approximate state-of-the-art in continuous-wave (CW) solid-state RF power systems. As seen for this figure, the CW power limit of solid-state devices is around 1 Watt at $100 \mathrm{GHz}$ and below $10 \mathrm{~W}$ over the entire millimeter-wave band. The technique to be described here will be shown theoretically to be able to produce power levels in excess of $10 \mathrm{~W}$ at these frequencies.

The generation of RF signals by the beating together of two optical signais, which is fundamental to the technique to be describe here, has been demonstrated by numerous researchers (e.g. [1]-[3]) and is even used in a commercial product from Anritsu[4]. Recently, there has even been a successful demonstration of RF generation at $250 \mathrm{GHz}$ by $\mathrm{E}$. R. Brown et al[5]. All of these systems produced low-power levels $(\mu \mathrm{W})$. We feel that this has occurred due to a lack of adequate laser power, difficulty with optical beam combination, and/or most importantly the lack of an adequate photodetector. All of these critical issues will be addressed in this report.

This paper provides a detailed development of the feasibility of RF generation by an optical means. The first section covers the necessary preliminary theory and develops the basis of the technique. This includes a simple development of the operation of photodiodes, and a discussion on the required optical signal. The next section describes the critical component of the system -- the traveling-wave photodetector. Finally, the last section brings together the concept with a hypothetical system example. 


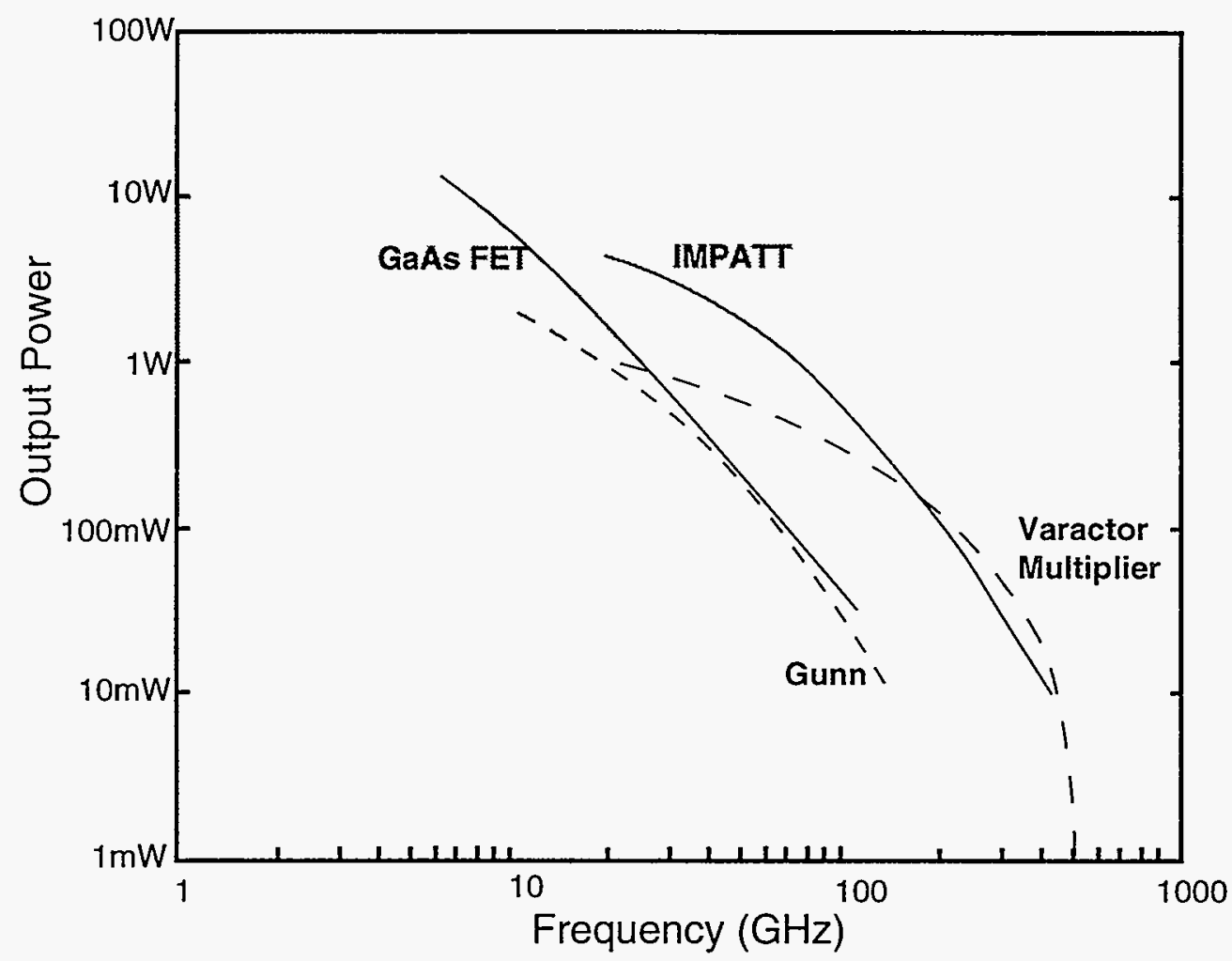

Fig. 1. State-of-the-art of solid-state RF power generation [6].

\section{Theory of Operation}

In this section, the theoretical basis for our technique is presented. The operation of photodetectors is first reviewed. The ideal modulated light signal is developed and two possible techniques for generation are described.

\section{Photodetector}

Photodetectors convert modulated light into electrical signals. Most solid-state photodetectors operate by incident light (photons) being absorbed which "creates" carriers (electrons and holes). These carriers either change the device's conductivity (i.e. photoconductors) or are swept away by an internal electric field to electrical contacts which produces a photocurrent (i.e. PINs, APDs). In either case, an electrical change occurs and light detection is achieved. A photodetector generally looks electrically like an ideal current source.

The most common measure of quality of the conversion of light to electrical current is the quantum efficiency. The quantum efficiency is the ratio of carriers generated to incident photons. That is, if for every photon incident on the device one electron-hole pair is generated, the detector is said to have a 
quantum efficiency of $100 \%$. Low-bandwidth photodetectors with quantum efficiencies approaching $100 \%$ (say $80 \%$ ), are not uncommon.

As background, it is instructive to develop a simple analytical expression for the responsivity $(\mathfrak{R})$ which is the photodetector's output current per incident optical power. The total optical energy $\left(E_{T}\right)$ incident on a detector can be expressed as:

$$
E_{T}=h f n
$$

where $h$ is Planck's constant $\left(6.63 \times 10^{-34} \mathrm{~J} . \mathrm{sec}\right), f$ is the optical frequency, and $n$ is the total number of incident photons. The instantaneous incident optical power $\left(P_{\text {opt }}\right)$ is then:

$$
P_{o p t}=\frac{d E_{T}}{d t}=h f \frac{d n}{d t} .
$$

The current in a photodetector $(I$ caused by the conversion of photons can be described as:

$$
I=\xi e \frac{d n}{d t}
$$

where $\xi$ is the quantum efficiency (ranges from 0 to 1 ), and $e$ is the charge of one carrier $\left(1.602 \times 10^{-19} \mathrm{C}\right)$. Eq. (3) states that the rate of incident photons $d n / d t$ produces a rate of charge carriers $\| / e$ with a probability $\xi$. Combining (2) and (3) gives:

$$
I=\frac{\xi e}{h f} P_{o p t}=\Re P_{o p t}
$$

where $\Re$ is termed the responsivity of the detector given in units of Amperes/Watt. From (4) and using $f=c / \lambda_{o p t}$ :

$$
\Re=\frac{\xi e}{h c} \lambda_{\text {opt }}
$$

where $c$ is the speed of light $\left(3 \times 10^{8} \mathrm{~m} / \mathrm{s}\right)$, and $\lambda_{o p t}$ is the optical wavelength.

At the wavelength to be used in the system example discussed in the last section of this report, $\lambda_{o p t}=838 \mathrm{~nm}$, and assuming $100 \%$ quantum efficiency $(\xi=1.0)$, (5) yields $\Re \approx 0.675 \mathrm{~A} / \mathrm{W}$. This means that $0.675 \mathrm{~A}$ of current would flow through a hypothetical $100 \%$ quantum-efficiency detector when $1 \mathrm{~W}$ of optical power is incident. Clearly, significant currents are feasible for realistic optical power levels. For an RF modulated optical source, and assuming that the detectors bandwidth is large (section 2.3 ), the peak RF current $\left(I_{p}\right)$ developed in the detector will be:

$$
I_{p}=\frac{I_{p-p}}{2}=\frac{\Re P_{o p r, p}}{2}
$$

where $I_{p-p}$ is the peak-to-peak current in the detector, and $P_{o p t, p}$ is the peak incident optical power. Given that the optical drive signal is modulated in a sinusoidal manner, the RF output power $\left(P_{R F}\right)$ is simply: 


$$
P_{R F}=\frac{1}{2} R_{o} I_{p}{ }^{2}=\frac{1}{8} R_{o} \Re^{2}\left(P_{o p t, p}\right)^{2}
$$

where $R_{0}$ is the resistance driven by the detector. This result is independent of frequency since the frequency response of the detector is assumed constant. In practice, all photodetectors will suffer from rolloff caused by electrical parasitics and from carrier transit time limits. The important result of (7) is that the RF output power depends as a quadratic function of the optical power.

Assuming $R_{O}=50 \Omega$ and using the responsivity found above, the graph shown in Fig. 2 illustrates the implications of (7). In this graph, the peak optical power range, $100 \mathrm{~mW}$ to $10 \mathrm{~W}$, was selected as a realistic estimate of powers available in the near future (over $0.4 \mathrm{~W}$ is presently available, see Section 5). Upon first inspection, the result plotted in Fig. 2 may appear flawed as conservation of energy appears to be violated. In fact, for 10 Watts of peak optical power ( $5 \mathrm{~W}$ average), over 280 Watts of RF power will be produced. This is the correct result. For the parameters used here, the RF output power will exceed the peak optical power when $P_{\text {opt, }}>351 \mathrm{~mW}$. Energy conservation is not violated. The detector's bias supply delivers the power to the RF output. This will be discussed in detail in the following section.

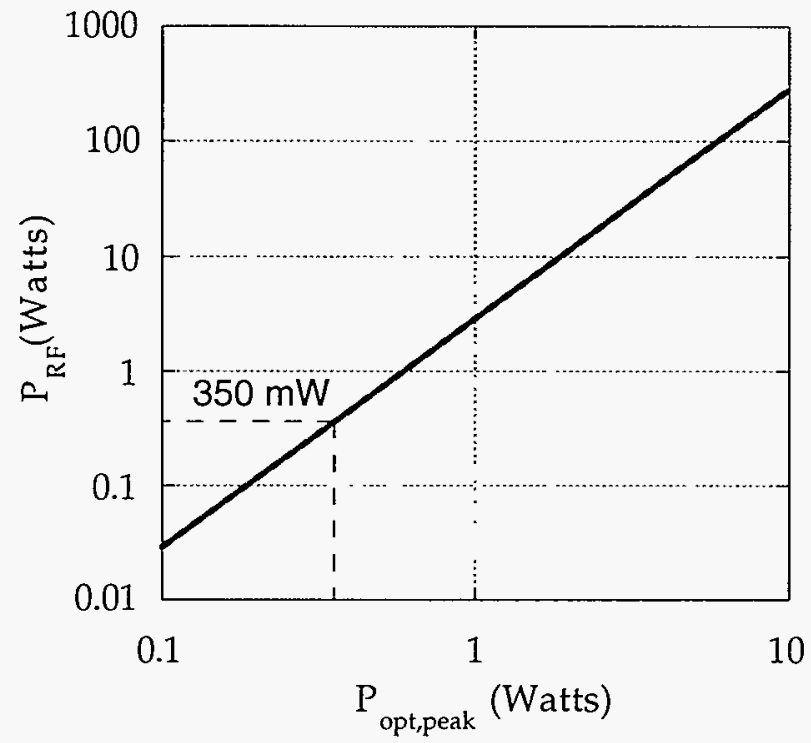

Fig. 2. RF output power verses peak optical power. Note that $P_{\text {opt,peak }}=4 P_{\text {laser }}$ for the dual laser system in Fig. 4. Dashed line shows were RF output power equals the optical input power.

The RF output power shown in Fig. 2 is also a function of the load resistance the detector is driving $\left(R_{0}\right)$. For the calculation of Fig. $2, R_{O}=50 \Omega$ was used. If instead this resistance was increased to $500 \Omega$, by (7), the RF output power would increase by a factor of 10 . This is not a surprising result as theoretically, a current source can deliver infinite power. The power obtained from an ideal current source is simply its current squared times the resistance it 
is applied to $(R R)$. Since a photodetector can be viewed electrically as an ideal current source and electrical impedance matching can be employed, this allows for additional flexibility in generating RF power.

\section{Conversion Efficiency}

As mentioned before, upon casual observation, there appears to be a contradiction in energy conservation with this technique. Here a brief discussion of the power efficiency of the photodetector is given. This discussion resolves this apparent contradiction and additionally provides added insight into component requirements for the system.

As used in the previous section and further to be assumed throughout this paper, the optical power incident on the photodetector is assumed to be sinusoidal. The sinusoidal power variation is assumed to vary from 0 to $P_{\text {opt,peak. }}$. Therefore, the incident optical power can be described analytically as:

$$
P_{\text {opt }}(t)=\frac{P_{\text {opt, peak }}}{2}\left(\cos \left(\omega_{R F} t+\Delta \theta\right)+1\right)
$$

where $t$ is time, $\omega_{\mathrm{RF}}$ is the angular RF operating frequency, and $\Delta \theta$ is an arbitrary phase offset. When incident on a photodetector, the resulting current is:

$$
I(t)=\Re \frac{P_{\text {opt peak }}}{2}\left(\cos \left(\omega_{R F} t+\Delta \theta\right)+1\right)
$$

where $\mathfrak{R}$ is the responsivity as defined in (4). Assuming that the output of the detector is capacitively coupled, only the AC term of (9) need be considered in determining the RF output voltage. The detector circuit's AC output voltage $\left(V_{\text {out }}\right)$ will be:

$$
V_{o u t r}(t)=R_{o} I(t)=R_{o} \Re \frac{P_{o p t, p}}{2} \cos \left(\omega_{R F} t+\Delta \theta\right) .
$$

From this, it is apparent that the peak-to-peak output voltage is simply:

$$
V_{o u t, p-p}=R_{o} \Re P_{o p t, p} .
$$

Throughout this paper the detector is assumed to be a semiconductor diode type which must be reverse biased. Additionally, it is assumed that the detector is biased with a reactive bias tee. Therefore, the detector's bias voltage must be, as a minimum, one-half of the peak-to-peak RF output voltage swing, or:

$$
V_{\text {bias }}=\frac{1}{2} R_{o} \Re P_{\text {opt,p }} .
$$

This value being $1 / 2$ the peak-to-peak RF output voltage may appear unusual, but the additional potential required to achieve the peak-to-peak output swing is "generated" by the reactive bias tee. An ideal reactive bias tee, which is for all 
practical purposes achievable at a given frequency, will look like an infinite inductance to the detector at the RF operating frequency. An infinite valued inductor will not allow any current change through itself $(I=L d V / d t)$. It effectively produces the voltage required to maintain its constant current. Therefore, as the current changes in the detector, the bias tee effectively converts in into the voltage (as described above) which appears across the RF load.

With (12) and the DC component of (9) the total power consumed from the DC detector bias supply must be:

$$
P_{\text {det }}=I_{\text {arg }} V_{\text {bias }}=\frac{R_{o}}{4} \Re^{2}\left(P_{o p t, p}\right)^{2}=2 P_{R F}
$$

where $l_{\text {avg }}$ is the DC term from (9) an the last equality is found from (7). With this result, several interesting and important observations can be made:

1.) The systems total average input power is:

$$
P_{i n}=P_{\text {det }}+P_{\text {opr,avg }}=2 P_{R F}+P_{\text {opr }, \text { avg }}
$$

where $P_{\text {in }}$ is the total input power, and $P_{\text {opt,avg }}$ is the average optical input power. With this the efficiency of the RF power generation is:

$$
\text { Efficiency }=\frac{P_{R F}}{P_{\text {in }}}=\frac{P_{R F}}{2 P_{R F}+P_{\text {opt.avg }}} .
$$

For large optical powers where $P_{R F}$ greatly exceeds $P_{o p t, a v g}$, a maximum system efficiency of $1 / 2$ or $50 \%$ could be achieved.

2.) The detector must be able to dissipate:

$$
P_{\text {det }}=P_{m}-P_{o m t}=P_{R F}+P_{c u t, u v g}
$$

If several Watts of RF power are output, the same amount of power plus the incident light's power must be dissipated. These sorts of power dissipation levels are simply unrealistic for traditional highbandwidth (small-size) photodetectors. The traveling-wave photodetector presented later in this report overcomes this problem.

Clearly, energy conservation is not violated in this system. The maximum efficiency of the system is in fact only $50 \%$. The power of the incident light is consumed in the generation of electron-hole pairs in the photodetector. These electrons and holes are then swept away from one another by the detectors bias supply to the detector's contacts. The electrons and holes gain energy from the bias supply as they are swept across the detector. To maintain charge neutrality, a current external to the detector must flow, resulting in RF output power. From this discussion, it is reasonable to say that all of the RF output power comes from the conversion of power from the detector's DC power supply. Therefore, there is no energy conservation contradiction. 


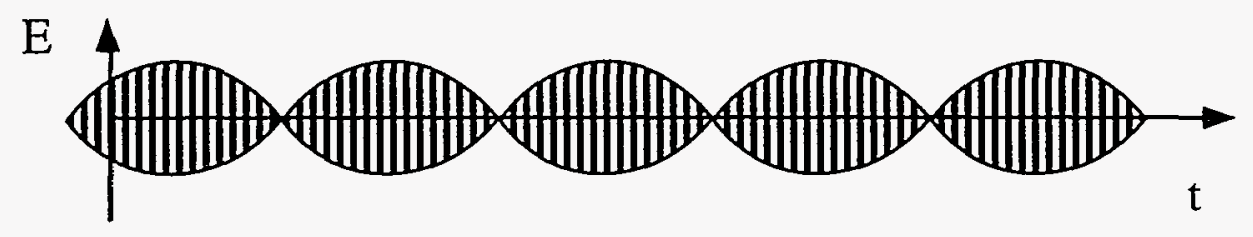

a)

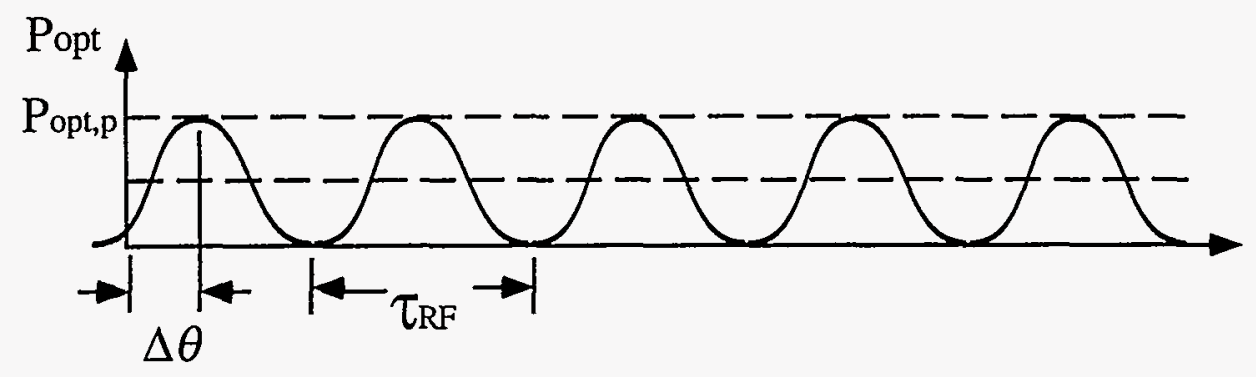

b)

Fig. 3. Ideal modulated light source for RF generation.

a) Electric field. b) Power envelope.

\section{Modulated Light Source}

As mentioned before and expressed analytically in (8), the optical power for this technique must vary sinusoidally between 0 and $P_{o p t, p}$. This is shown graphically in Fig. 3b. Such a power envelope is the logical choice for this application. Clearly, the selected power envelope must have a sinusoidal time dependence, as a sinusoidal RF signal is desired at the output. Secondly, the power envelope is logically selected to range from zero upward, as one desires to minimize the DC component of the optical signal in-order-to minimize the detector's DC power dissipation. Finally, besides appearing to be the ideal optical power envelope, it is relatively easy to generate.

Two techniques for the generation of this optical signal will be presented. The first technique is based on the beating together of two CW lasers and the second, on the use of a single laser and an external modulator. Both techniques have their own unique merits and disadvantages. The techniques given here are not inclusive of all techniques, as there are undoubtedly numerous other possibilities (i.e. mode-locked laser sources).

\section{Dual Light Source}

The simplest approach for the generation of the required optical signal is to beat together two coherent optical sources. Such a system is shown schematically in Fig. 4. The coherent sources are assumed to be singlefrequency lasers. The two lasers are equal in power and are slightly "detuned" from one another in-order-to produce the required beat frequency. The outputs of the two lasers are combined and directed to the photodetector. Such a technique is commonly used to test the electrical bandwidth of photodetectors[7]. 


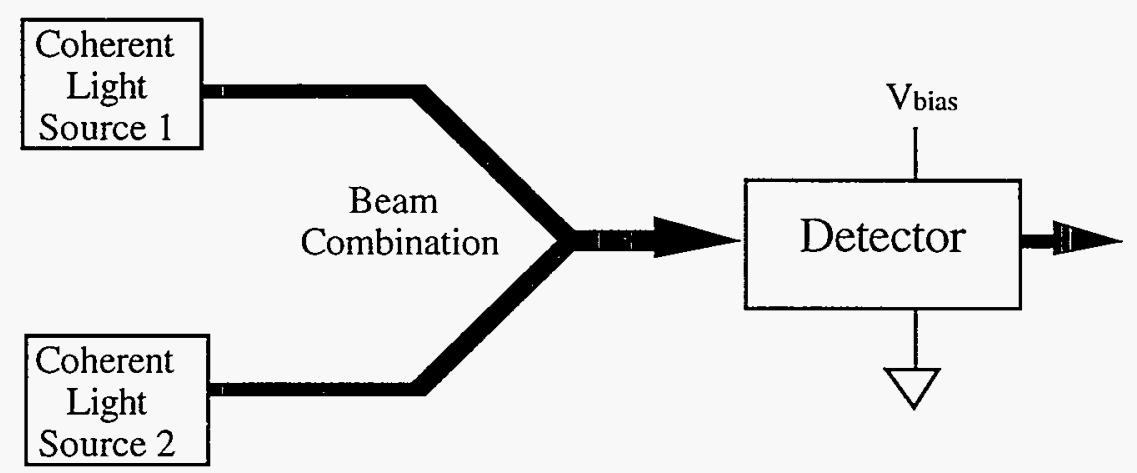

Fig. 4. Dual light source system.

Combination of the two light beams may pose an interesting problem. Free-space optic combination approaches may be possible, provided great care is taken for phase preservation of the optical wavefronts. Phase preservation is critical due to the fact that, when heterodyning, any phase shift introduced into one of the optical signals will map directly into the phase of the RF output. Therefore, if the optical phase front varies across the surface of a detector, generated photocurrents will very likely combine destructively in part or in whole. This will result in a reduction (or possible total elimination) of RF output. One approach to avoid this problem is to use single-mode optics. If both lasers are coupled into single mode fibers and combined with a standard $-3 \mathrm{~dB}$ coupler, the resulting optical signal sent to the photodetector will be in proper phase relationship. The only draw-back with such an approach is the inevitably large losses introduced by coupling in and out of the fiber (several $\mathrm{dB}$ per connection) and the loss within the coupler itself. Free-space combination schemes may offer higher efficiencies and additionally, may be usable with the traveling-wave photodetector discussed in the next section.

By far the most difficult aspect of using two lasers to produce the required RF beat frequency is in controlling the wavelength offset between the two lasers. Since the frequency $(f)$ is related to wavelength $(\lambda)$ by $f=c / \lambda$ where $c$ is the speed of light, the required wavelength offset $\left(\Delta \lambda_{0}\right)$ is:

$$
\Delta \lambda_{o}=\frac{ \pm\left(f_{R F} / c\right) \lambda_{o}}{1 \pm\left(f_{R F} / c\right) \lambda_{o}} \approx \pm\left(f_{R F} / c\right) \lambda_{o}= \pm \frac{\lambda_{o}}{\lambda_{R F}}
$$

where $f_{R F}$ and $\lambda_{R F}$ are the RF beat frequency and wavelength, respectively; and $\lambda_{o}$ is the optical wavelength. Since the RF wavelength is many times larger than the optical wavelength, the required optical wavelength shift is very small. For example, at $\lambda_{o}=840 \mathrm{~nm}$ and $f_{\mathrm{RF}}=60 \mathrm{GHz}, \Delta \lambda_{o} \approx 0.000168 \mathrm{~nm}$.

Such a small laser wavelength difference certainly poses control difficulties. Figs. 5 and 6 show two possible techniques to control this small wavelength offset. In both approaches, the lasers' outputs are assumed to be isolated from one another. If they are not well isolated, the lasers may "injection lock" to the same wavelength. The approach shown in Fig. 6 uses injection 
locking of the two lasers to an frequency offset sample of one of the lasers. Note that the outputs must still be isolated from one another.

The approach in Fig. 5, uses a physical means (vs. optical coupling) to control the operating wavelength of the two lasers. Possible examples of this physical control include mechanical size change by piezo-electric means or temperature. Temperature tuning of the operating wavelength of a laser is a common approach for wavelength control. The commercial single-frequency $100 \mathrm{~mW}$ CW $840 \mathrm{~nm}$ laser discussed in this report's last section, has a temperature tuning sensitivity of $0.067 \mathrm{~nm} /{ }^{\circ} \mathrm{C}$ at room temperature. Therefore, for the $\Delta \lambda_{o}$ given above, the required $\Delta T$ becomes $0.000168 \mathrm{~nm} / 0.067 \mathrm{~nm} /{ }^{\circ} \mathrm{C}$ or $0.00251^{\circ} \mathrm{C}$. This is indeed a small very temperature offset, but it is achievable in a controlled fashion.

The frequency discriminator circuit/network shown in Fig. 5 could be used to monitor the output frequency difference. The output from the frequency discriminator would be fed back to the temperature controller to form a control loop. Note that only a temperature difference is required; rather than an exact temperature. This greatly simplifies the control requirements.

Above we have tacitly assumed that the two lasers are identical. Two lasers are rarely only $0.000168 \mathrm{~nm}$ apart in wavelength at a given operating temperature and power level. One could, though, envision, two lasers produced side-by-side on the same monolith with very similar properties. If this were the case, it is very likely that the required $\Delta T$ would be similar to that predicted above. Under such circumstances, the required $\Delta T$ could be achieved by attaching the dual-laser chip to a thermally conductive bar. The ends of this bar would be held at appropriate temperatures to achieve the required temperature offset. The bar forms the analogy of a simple lever used to lower the sensitivity of the tuning.

Simple "open-loop" temperature tuning for controlling the beat frequency between two lasers suffers from large RF linewidth. The RF output linewidth will be similar to the laser's optical linewidth (1/2 power width). The linewidth of solid-state lasers vary greatly depending on their type. For example, with Nd:YAG diode pumped lasers, the linewidth may be as narrow as $10 \mathrm{KHz}$. With GaAs/AIGaAs single-stripe edge-emitting lasers (see section 4.0) the linewidth may be as wide at $10 \mathrm{MHz}$. For some RF applications, this may be acceptable. For other applications, it may be very undesirable. In principle, it is possible to form a closed-loop electrical feedback system which would allow for very narrow linewidths provided some means of fast electrical laser wavelength control is available. This fast frequency control could possible be achieved by the diode current. If the cooler/heater temperature is the only control available, the resulting RF linewidth will match the laser's linewidth.

The primary benefit of the dual laser approach is in the optical output power achieved. Since two lasers are used and further no optical manipulation is required (except to combine their beams), a large peak optical power is achievable. Using the notation developed in section 2.1, the peak optical power $\left(P_{\text {opt }, p}\right)$ is: 


$$
P_{\text {opt }, p}=4 P_{\text {laser }}
$$

where $P_{\text {laser }}$ is the power of an individual laser. The peak optical output power is four times that from an individual laser. Note that (18) indicates that the average optical output power is $2 P_{\text {laser }}$ exactly the power emitted from the two lasers.

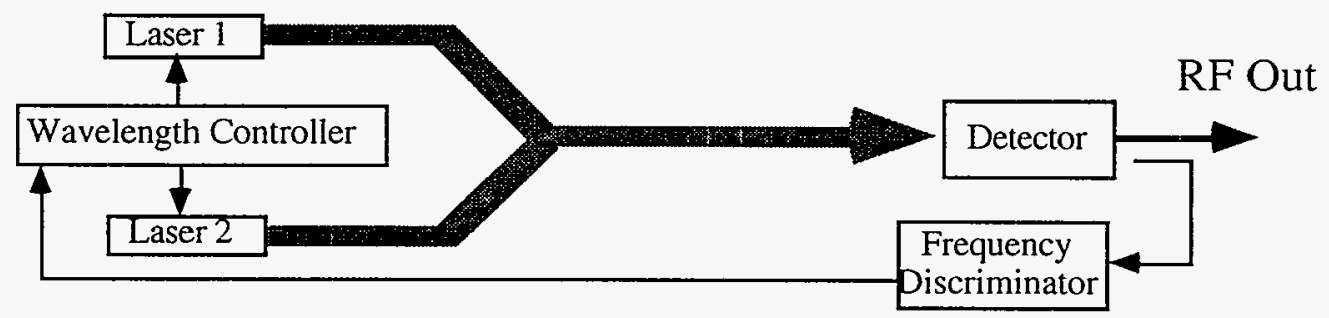

Fig. 5. Wavelength control of dual light source system.

Fig. 6 illustrates another possible technique for wavelength control. Here the lasers are locked together by injecting a locking signal from one laser into the other. The lower laser is termed the master and the upper the slave. The slave laser is wavelength referenced to a wavelength offset version of the master laser. Hence, the master laser determines the operating wavelength of both lasers. The slave laser simply follows a frequency offset version of the master laser. In the figure, the lower master laser operates at some optical frequency $\omega_{0}$. A sample of its output is directed to an SSB modulator which frequency translates it. The optical signal is then injected into the slave laser for locking. The output from the two lasers is again combined and sent to the photodetector.

This technique offers the appeal of producing a very narrow RF linewidth. Since the two lasers are locked together, any wavelength fluctuations which appear in the master laser will similarly appear in the slave laser. Then, the RF output beat frequency will remain constant. The down-side of this technique is that it requires an SSB modulator which can operate at the desired RF frequency. The only commercially available device which can perform the required frequency translation is an acousto-optic modulator[8]. Such modulators are unfortunately usable only up to frequencies of a few $\mathrm{GHz}$.

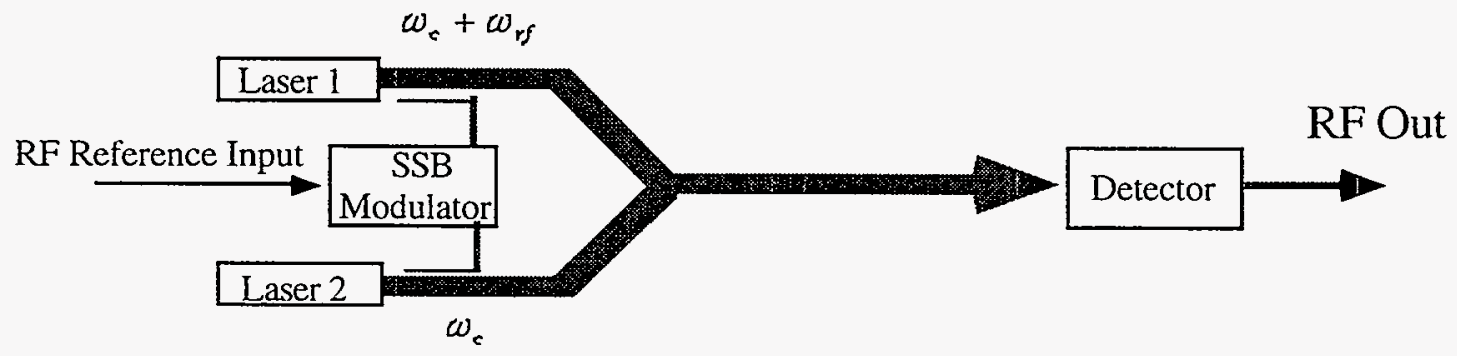

Fig. 6. Injection locking of dual light source system. 


\section{Single Light Source}

Another approach to generating the necessary optical drive signal is to use a single modulated light source. This modulation may be internal (modulation of laser current or mode locking) or external (modulation external to laser). Ether way, the obvious disadvantage of this approach is that at maximum efficiency only $1 / 2$ the optical power will be available for a single verses a dual laser system. This will reduce the RF power to $1 / 4$ a dual laser system. Since only one laser is used, the advantage of this approach is that a narrow RF linewidth can be obtained.

A single light source system is shown in Fig. 7. A single coherent light source (laser) is modulated by an external modulator and then detected by a photodiode. If an appropriate large-bandwidth high-power laser diode existed, one could directly modulate the laser and avoid the need for an external modulator.

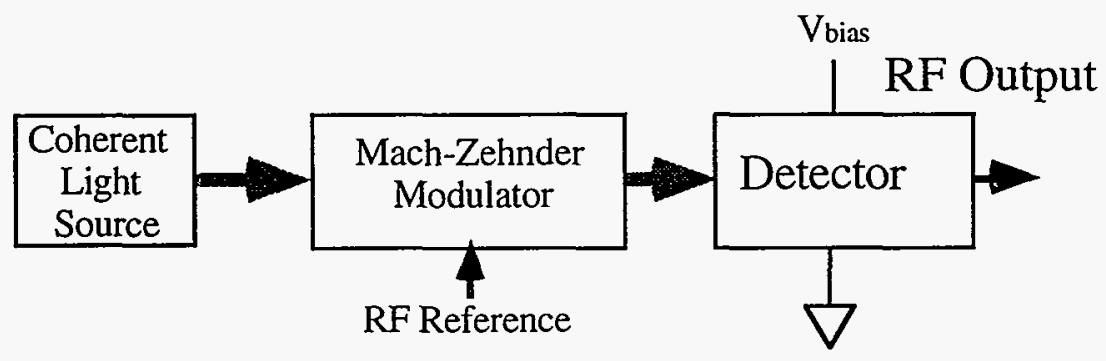

Fig. 7. Single light source system.

\section{Component Requirements}

To achieve a useful RF power level using this technique, several components are required. First, a light source with the output as described above with appropriate power levels must exist. Secondly, a photodetector which has appropriate bandwidth, efficiency, and power dissipation capabilities is required.

The optical source must be capable of producing peak optical powers on the order of 1 Watt to be competitive. As was seen in Fig. 2, at these power levels several Watts of RF power should be possible. A hypothetical system using $100 \mathrm{~mW}$ commercial lasers which develops a peak optical power of $0.4 \mathrm{~W}$ will be discussed in the last section of this report.

A suitable photodetector is more difficult to obtain. Large-bandwidth photodetectors with good responsivity do exist [9], but they are all physically small and thus unable to handle the incident optical power or bias power levels required. For this technique to be realized, a new photodetector must be developed. The critical feature of this detector be that it must be able to dissipate large power levels. This suggests that the detector must be large. to reduce the power density of light absorbed inside the device, while still 
maintaining suitable electrical bandwidth. The traveling-wave photodetector discussed in the next section satisfies this requirement. Large RF power levels from such optical systems have not been observed to date due to this lack of suitable photodetectors.

\section{Traveling-wave Photodetector}

This section describes a photodetector which is capable of dissipating the large power levels discussed above. Large power dissipation levels are achieved by the detector's large size. The large size with simultaneous large bandwidth, and high-quantum efficiency is only possible with a traveling-wave design. The basic theory of operation of the photodetector is presented in this section.

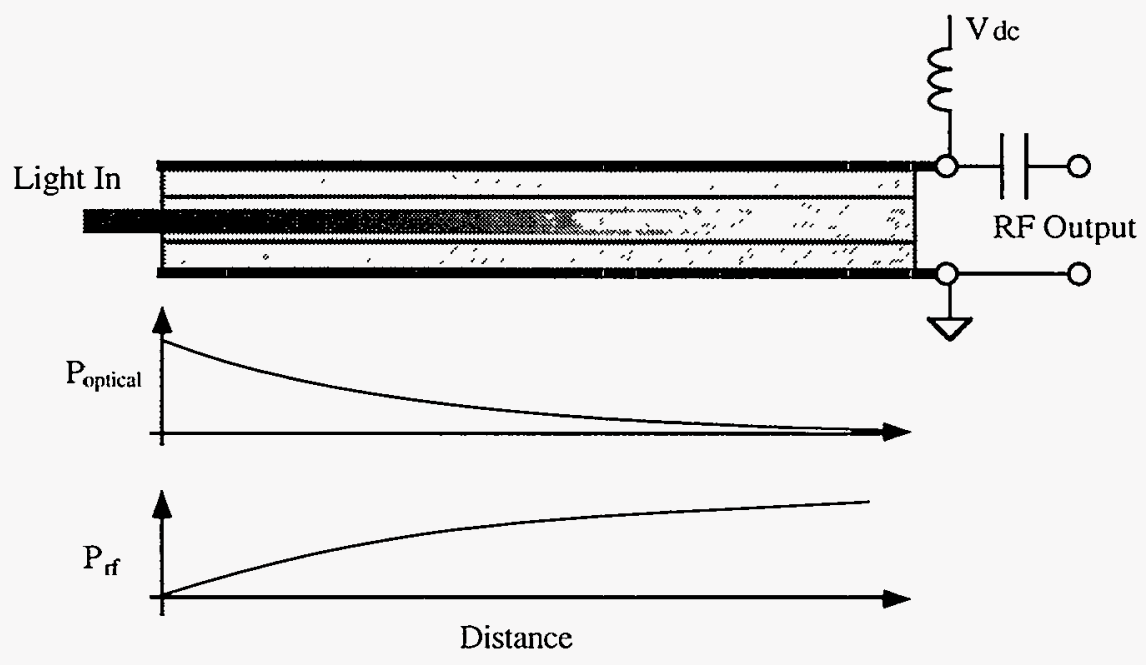

a)

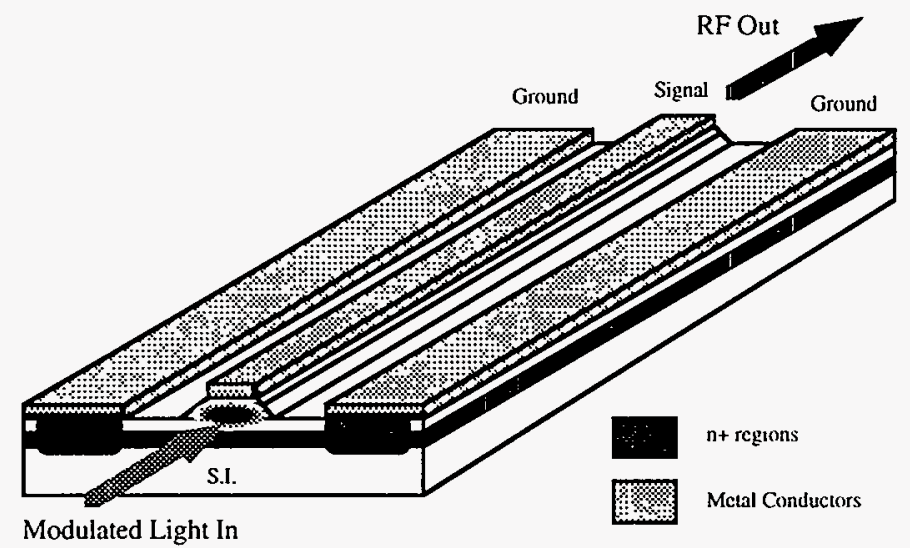

b)

Fig. 8. Diagram of traveling-wave photodetector. a) Optical and RF power levels are shown along the detector's length. b)Physical diagram. 
Traditional photodetectors are electrically lumped devices. That is, they can be considered to exist at one point in space where all of the photogeneration takes place. This means that the electrical bandwidth of traditional detectors is $R C$ limited $\left(f_{-3 d B}=1 /(2 \pi R C)\right)$. Therefore, one strives to minimize the device's capacitance $C$ by reducing the detector's size. This has the unfortunate side effect of reducing detectors responsivity and the maximum power dissipation. The traveling-wave photodetector (TWPD) overcomes this limitation by being an electrically distributed device.

\section{Theory of Operation}

Fig. 8 illustrates the fundamental operation of the traveling-wave photodetector. The photodetector can be made physically large by controlling the optical absorption in the detector and by designing its electrode structure for velocity synchronism with the light. Light is launched into an optical waveguide formed in the junction of a PIN diode (GaAs/AIGaAs in present design). The thickness of the absorbing region in the PIN diode is adjusted to set the absorption length in the waveguide. Use of a thin layer $(\approx 100 \AA$ for GaAs @ $1.06 \mu \mathrm{m}$ ) permits for a very large absorption length. The absorption is selected to be high enough for the majority of the light (say 99\%) to be absorbed for high quantum efficiency, while still being low enough to allow for a large device length to achieve high power dissipation. Light is "detected" in an extended region along the PIN diode. This creates distributed current generation along the detector's length. The metal electrodes are designed to allow for this generated current (RF signal) to "travel" in synchronism with the light. Hence the name traveling-wave photodetector. If velocity synchronism is achieved, the photo-generated distributed current will add at the detector's output.

This photodetector exhibits a high quantum efficiency and a large maximum power dissipation due to its large size. Additionally, its bandwidth should be primarily determined by the electrical bandwidth of the metal electrode structure. The electrode structure employed is a slow-wave coplanar transmission line, of which a similar transmission line has been demonstrated elsewhere and exhibited useful operation to $40 \mathrm{GHz}[10]$.

A detailed model for detection along the length of this detector has been developed. This model was developed during the early part of this project [11] and is similar to a time-domain model described recently by Giboney et al [12].

Assuming no back reflection at the far end of the device (100\% optical absorption), and excitation by two equal-power $\left(P_{0}\right)$ lasers offset by the RF operating frequency, the optical intensity $\left(P_{l}\right)$ within the photodetector can be described by:

$$
P_{l}(z, t)=2 P_{o}\left(1+\sin \left(\omega_{r f} t-\beta_{o} z\right)\right) e^{-\alpha_{o} z}
$$

in which $\omega_{r}$ is the angular if beat frequency, $z$ is the position along the length of the detector, $\alpha_{0}$ attenuation constant of the optical mode and $\beta_{0}$ is the 
propagation constant of the if modulated light $\left(\beta_{O} \approx 2 \pi n_{d} \lambda_{r}\right)$. Dropping the $\mathrm{DC}$ term, (19a) can be rewritten in phaser-wave notation at the RF-operating frequency $\omega_{\text {rr.: }}$

$$
p_{l}(z)=2 P_{o} e^{-\gamma z}
$$

in which $\gamma=\alpha_{o}+\beta_{O}$. The exponential decay of the light as it propagates is due to photons being absorbed and hence electrons and holes generated (e.g. a current). The local current generated at any point along the detector is related to the amount of light absorbed at the point multiplied by the responsivity:

$$
\frac{d I_{d}(z)}{d z}=\Re \frac{d\left|p_{l}(z)\right|}{d z} e^{-j \beta z}
$$

in which $(\Re)$ is the responsivity given by:

$$
\Re=\frac{\xi_{e} \lambda_{o}}{h c}
$$

where $\xi$ is the quantum efficiency, $e$ is the charge of an electron, $h$ is Planck's constant, and $c$ is the speed of light. Carrier transit times within the detector are neglected allowing for $(20)$ to be valid at all operating frequencies. Combining (19) and (20) gives:

$$
\frac{d I_{d}(z)}{d z}=k_{o} e^{-\gamma_{o} z}
$$

in which

$$
k_{o}=\frac{\alpha_{o} \xi e P_{o} \lambda_{o}}{2 h c}
$$

and

$$
\gamma_{o}=\alpha_{o}+j \beta_{o} .
$$

This distributed current source can be coupled to the electrical propagation along the detector by incorporating it into the time-harmonic telegraphist's equations [13]:

and

$$
\frac{d V}{d z}=-Z I
$$

$$
\frac{d I}{d z}=-Y V-\frac{d I_{d}(z)}{d z}
$$

in which the second term is the differential current source described by (21). The transmission line equivalent circuit is shown in Fig. 9. This model is only approximately correct as physically the current source should be in parallel with the capacitance. The incorporation of the current source as shown in Fig. 10 is appropriate provided that the transverse electrical loss $\left(R_{T}\right.$ in (34)) is small compared to the electrical energy storage along the detector. This is true for 
this type of transmission line. Using this approximate model allows for an analytical solution. Eq. (22) can be combined to form a second order differential equation describing the voltage $(V)$ along the line:

$$
\frac{d^{2} V}{d^{2} z}-\gamma^{2} V=Z k_{o} e^{-\gamma_{o} z}
$$

in which $\gamma=\sqrt{Z Y}$ is the traditional electrical propagation constant along the detector.

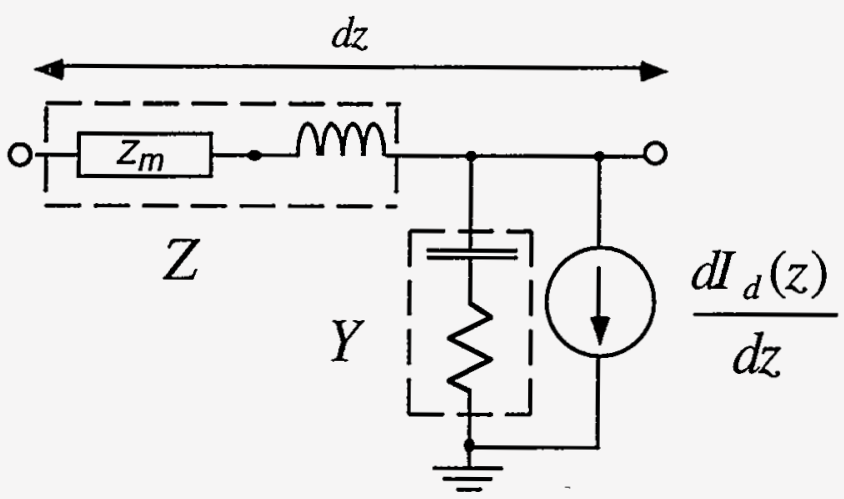

Fig. 9. Equivalent transmission-line circuit for TWPD.

Eq. (23) has a simple analytical solution which can be found by elementary methods (i.e. variation of parameters):

$$
V(z)=V_{1} e^{\gamma z}+V_{2} e^{-\gamma z}+\frac{Z k_{o}}{\left(\gamma_{o}^{2}-\gamma^{2}\right)} e^{-\gamma_{o} z} .
$$

The first and second terms in the right hand side of (24) are the traditional backward and forward propagating waves along a transmission line. The third term the detected light's signal impressed along the line. Applying boundary conditions allows for the determination of $V_{1}$ and $V_{2}$.

There are two sets of boundary conditions which are informative to apply to (24). First, allowing the input end (where the light enters the device) of the detector to be open with the output end (opposite the input end) terminated, as shown in Fig. 8. And secondly, with both the input and the output ends terminated.

With the input open and output ends terminated, the appropriate boundary conditions are:

$$
I(0)=0 \quad \text { and } \quad \frac{V(l)}{I(l)}=Z_{l} .
$$

Applying these boundary conditions to (24) gives:

$$
V_{1}=\frac{k_{o}\left(\gamma\left(Z_{l} \gamma_{o}-Z\right) e^{-\gamma_{o} l}-\gamma_{o}\left(Z_{l} \gamma-Z\right) e^{-\gamma l}\right)}{\gamma\left(\gamma_{o}^{2}-\gamma^{2}\right)\left[\left(1-\frac{Z_{l}}{Z_{o}}\right) e^{-\gamma l}+\left(1+\frac{Z_{l}}{Z_{o}}\right) e^{\gamma}\right]}
$$


and

$$
V_{2}=\frac{k_{o}\left(\gamma\left(Z_{l} \gamma_{o}-Z\right) e^{-\gamma_{o} l}-\gamma_{o}\left(Z_{l} \gamma+Z\right) e^{\gamma l}\right)}{\gamma\left(\gamma_{o}{ }^{2}-\gamma^{2}\right)\left[\left(1-\frac{Z_{l}}{Z_{o}}\right) e^{-\gamma l}+\left(1+\frac{Z_{l}}{Z_{o}}\right) e^{\gamma l}\right]}
$$

in which $Z_{0}$ is the characteristic impedance of the detector $(\sqrt{Z / Y})$ The RF output power from the detector will be the power developed in the output load $Z$.

$$
P_{r f}=\frac{|V(l)|^{2}}{2 Z_{l}} .
$$

Fig. 10 shows a calculation of if output power as a function of frequency for several different optical absorption lengths using the open input boundary condition (Eq. 25). In this calculation the detector has a $50 \Omega$ characteristic impedance, is infinite in length, and has a perfect velocity match between the electrical signal and the light. Other physical parameters used in the calculation are shown in Table 1. All normalized responses in this report are normalized to a conventional lumped photodetector of equal quantum efficiency, infinite bandwidth, and driving a $50 \Omega$ load. Interestingly, there is a $6 \mathrm{~dB}$ rolloff in the traveling-wave detector's response which has a characteristic frequency determined by the value of the optical absorption $\left(\alpha_{o}\right)$. This rolloff occurs at the transition frequency where the detector effectively becomes distributed. The $-3 \mathrm{~dB}$ point occurs when $\beta_{\mathrm{O}}=\alpha_{\mathrm{O}} / \sqrt{2}$ or equivalently at:

$$
f_{t}=\frac{\alpha_{o}}{2 \pi} \frac{v_{g}}{\sqrt{2}}
$$

where $v_{g}$ is the optical velocity along the guide. At low frequencies, the detector's response is identical to a lumped detector ( $0 \mathrm{~dB}$ in Fig. 10). The $6 \mathrm{~dB}$ rolloff suggests that the "effective" photocurrent is being halved or equivalently the detectors responsivity (or quantum efficiency) is being halved. Since the detector's DC quantum efficiency will approach $100 \%$ the effective quantum efficiency at high frequencies should approach $50 \%$.

\begin{tabular}{|c||c|}
\hline$Z_{O}$ & $50 \Omega$ \\
\hline$Z_{1}$ & $50 \Omega$ \\
\hline$n_{R F}$ & 3.5 \\
\hline$\alpha_{R F}$ & 0 \\
\hline$n_{O}$ & 3.5 \\
\hline$I$ & $\infty$ \\
\hline
\end{tabular}

Table 1. Parameters used for TWPD calculations. 


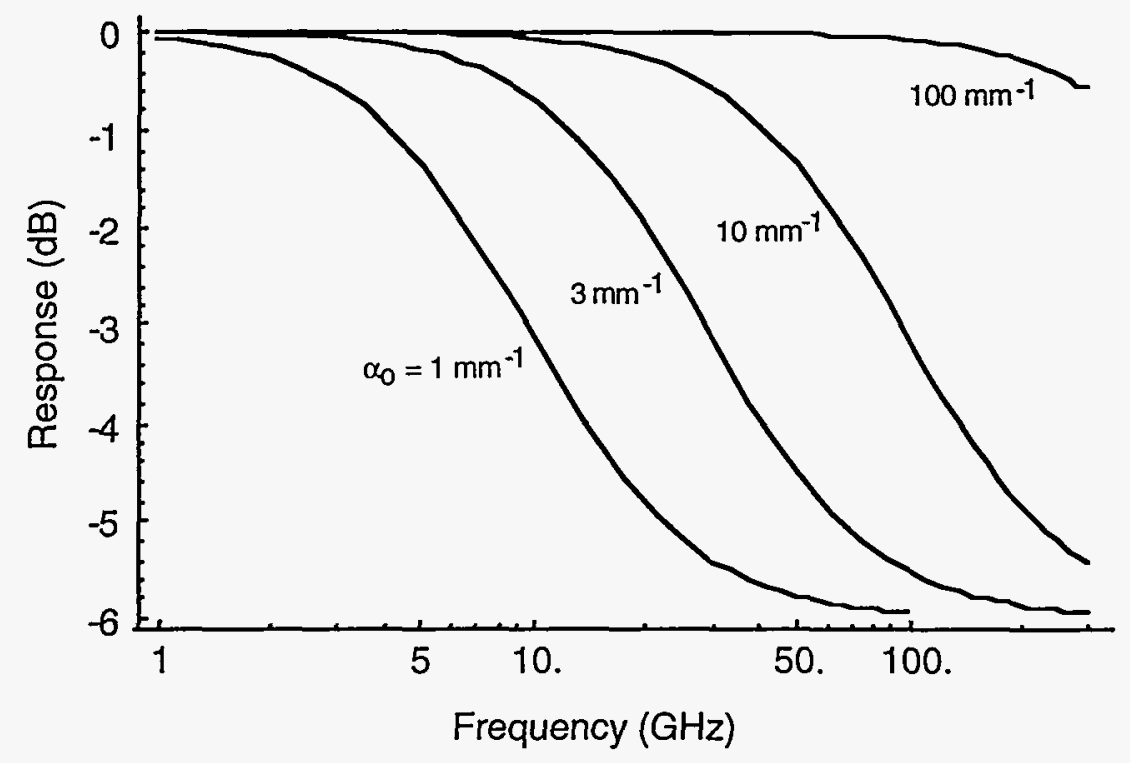

Fig. 10. Theoretical TWPD response with output terminated in $Z_{0}$ calculated for several different optical absorption values.

Terminating both the input and output ends of the detector provides additional insight. If both ends of the device are terminated in $Z_{0}$ (maximum power transfer), the necessary boundary conditions are:

Applying this to (23) gives:

$$
\frac{V(l)}{I(l)}=-\frac{V(0)}{I(0)}=Z_{o} .
$$

$$
V_{1}=\frac{k_{o}\left(Z+\gamma_{o} Z_{o}\right)}{2\left(\gamma^{2}-\gamma_{o}^{2}\right)}
$$

and

$$
V_{2}=\frac{k_{o}\left(Z+\gamma_{o} Z_{o}\right)}{2\left(\gamma^{2}-\gamma_{o}^{2}\right)} e^{-\left(\gamma_{o}+\gamma\right) \ell} .
$$

Fig. 11 shows a calculation of normalized detector response as a function of frequency for several different optical absorption lengths using the boundary conditions set by (29). The same parameters are used as were in the calculation of Fig. 10. Here, a similar rolloff in response is seen except only $3 \mathrm{~dB}$ reduction in signal occurs at high frequencies. This is at the expense of a $3 \mathrm{~dB}$ reduction in low frequency response as compared to the single termination case. The $3 \mathrm{~dB}$ reduction in response at low frequencies is consistent with applying two $Z_{o}$ terminations in parallel to a lumped detector. Interestingly, the high-frequency response approaches the same value as the single termination case. 


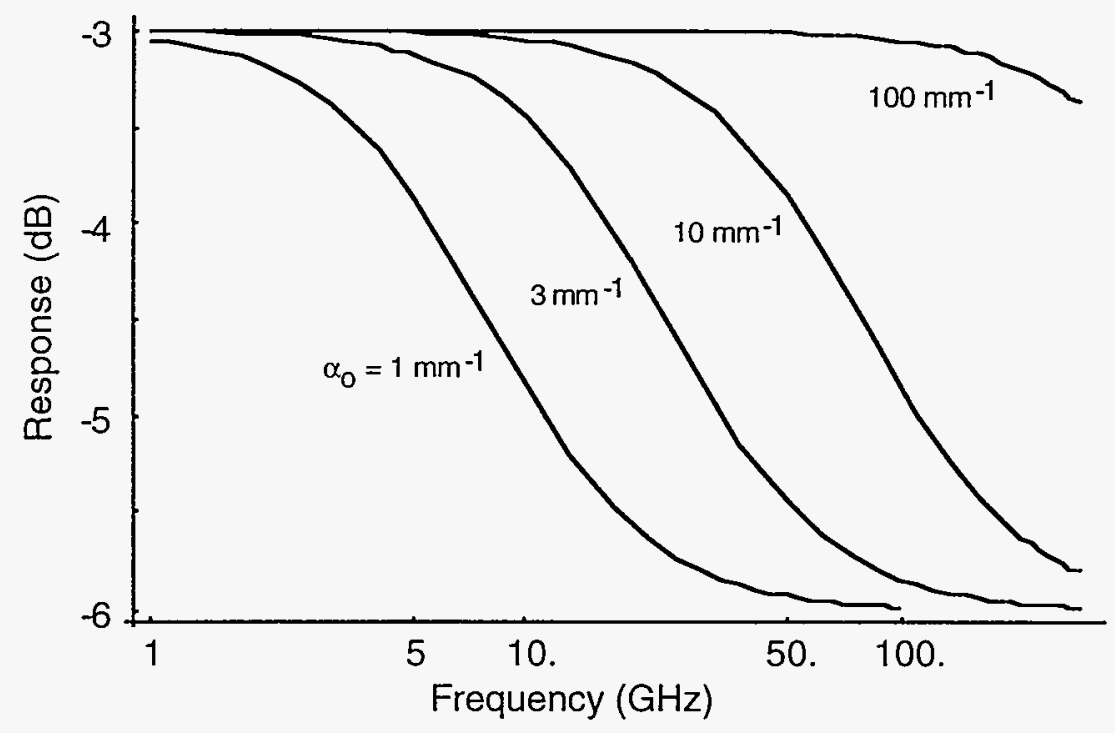

Fig. 11. Theoretical TWPD response with both input and output terminated in $Z_{0}$.

The response curves plotted in Fig. 11 include the power delivered to both terminations. It is informative to plot the response of the signal delivered to both terminations separately. Fig. 12 shows the response of the signals from either port when $\alpha_{0}=1 \mathrm{~mm}^{-1}$. Interestingly, the signal on the input termination $P_{R I}$ rolls off at a rate of $20 \mathrm{~dB} /$ decade like a simple $R C$, whereas the signal on the output termination is frequency independent. There is no signal at the input side of the detector at high frequencies. This explains why the single termination and dual termination solutions approach one another at high frequencies. The important result of the dual termination case is that a frequency independent response can be obtained by terminating both sides of the detector and only using the signal from the detector's output side.

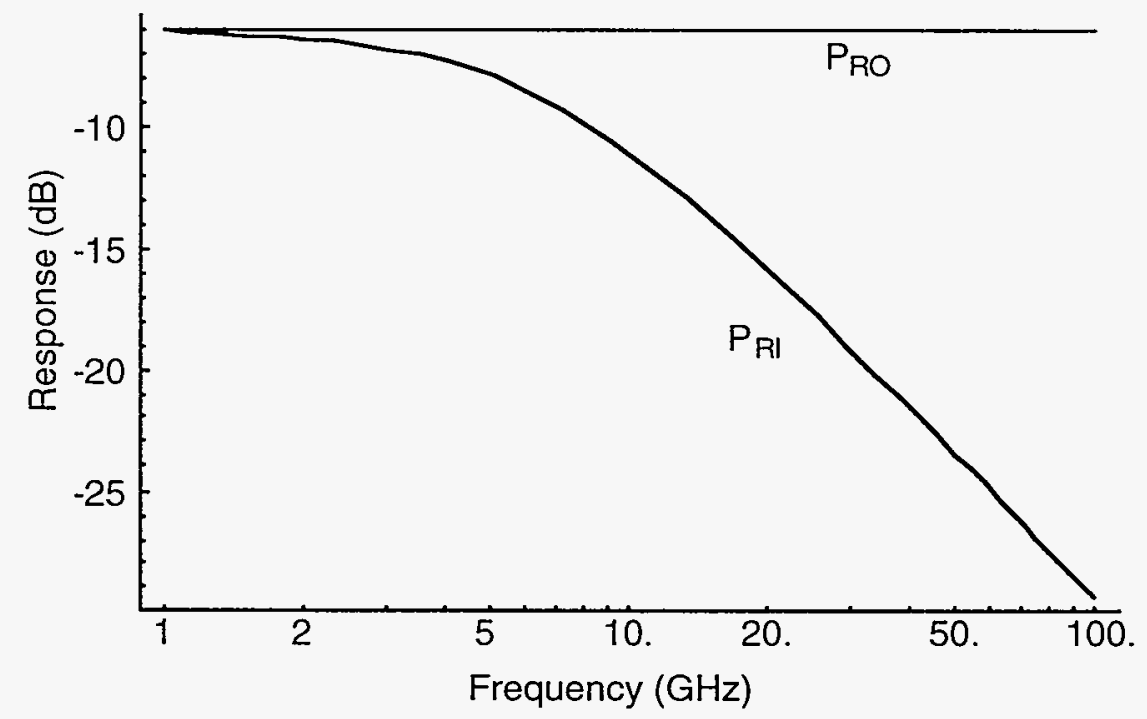

Fig. 12. Theoretical TWPD response with $\alpha_{O}=1 \mathrm{~mm}^{-1}$ for both input load $P_{R I}$ and output load $P_{R O}$. 


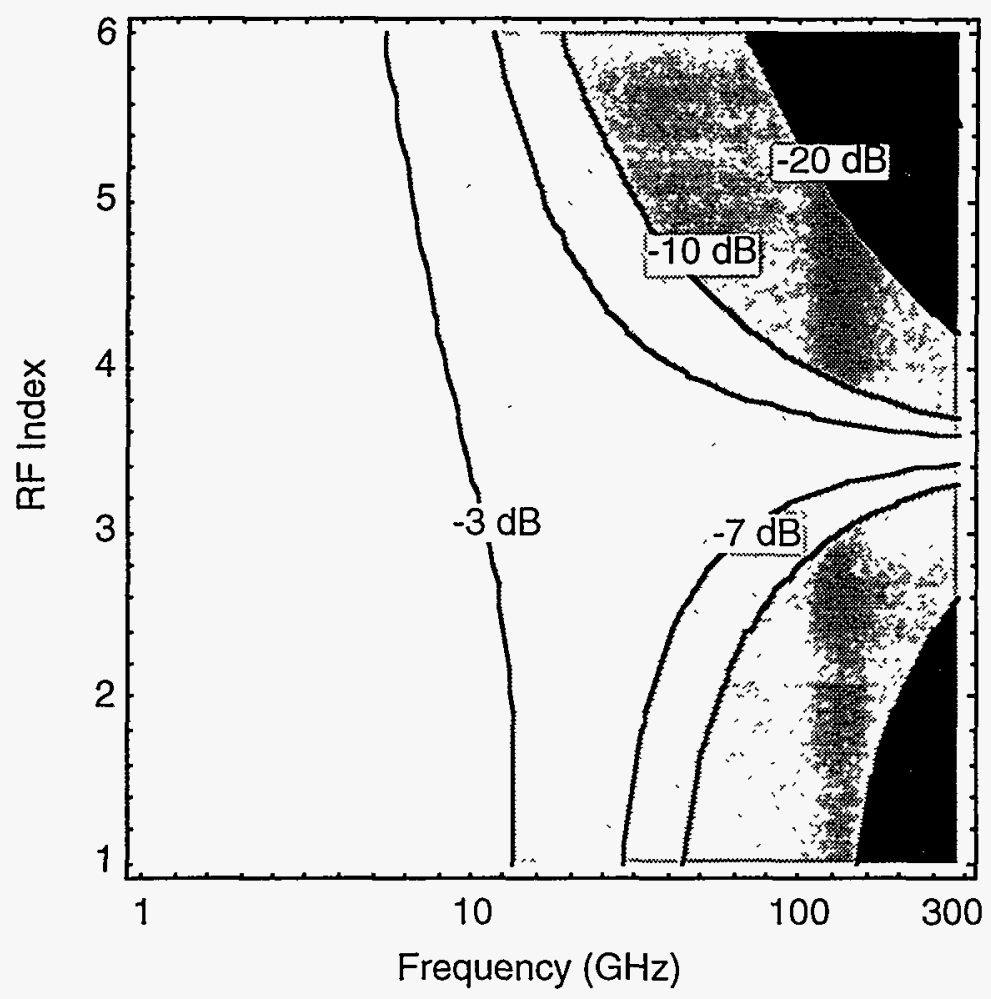

Fig. 13. Theoretical TWPD response as function of RF index and frequency. Parameters are given in Table 1 and $\alpha_{0}=1 \mathrm{~mm}^{-1}$.

To this point, all simulations have assumed velocity match. The model developed here is quite general and arbitrary velocity mismatches can be analyzed. Fig. 13 shows a contour plot of normalized response as a function of frequency and RF index. The optical index was fixed at 3.5 and the detector was terminated only on the output end. The RF index $\left(c / v_{p}\right)$ is plotted from the speed of light $\left(n_{r}=1\right)$ to $1 / 6$ the speed of light $\left(n_{r}=6\right)$. Slicing Fig. 13 along $n_{r f}=$ 3.5 produces the $\alpha_{0}=1 \mathrm{~mm}^{-1}$ curve in Fig. 10. At low frequencies where the electrical wavelength exceeds $1 / \alpha_{o}$, the RF index has little effect on the device's response since it's effectively electrically lumped. At very high frequencies where the wavelength is much less than $1 / \alpha_{0}$, the velocity match becomes critical as expected. Clearly, large high-frequency traveling-wave photodetectors need be velocity matched.

All calculations to this point have assumed no RF loss along the detector. In the following section a model which includes loss is developed for RF propagation along the detector is developed. This model is then used to predict response with RF losses included. 


\section{Electrode Design}

The optical waveguide and the electrode must be designed for a velocity match. The propagation velocity of light in the waveguide is fixed by its dimensions and material composition. In a GaAs/AlGaAs material system the optical index will always be approximately 3 . The actual optical velocity is not particularly critical since the RF-propagation velocity can be varied over a large range for synchronism. The metal electrode structure comprised of metal on top of the optical waveguide and ground planes to either side along with the "buried" heavily doped $\mathrm{N}+$ layer, forms a slow-wave coplanar transmission line (see Fig. 14). The electrical propagation velocity of this transmission line is tunable over a large range by varying the line's dimensions. Hence, velocity synchronism can be achieved.

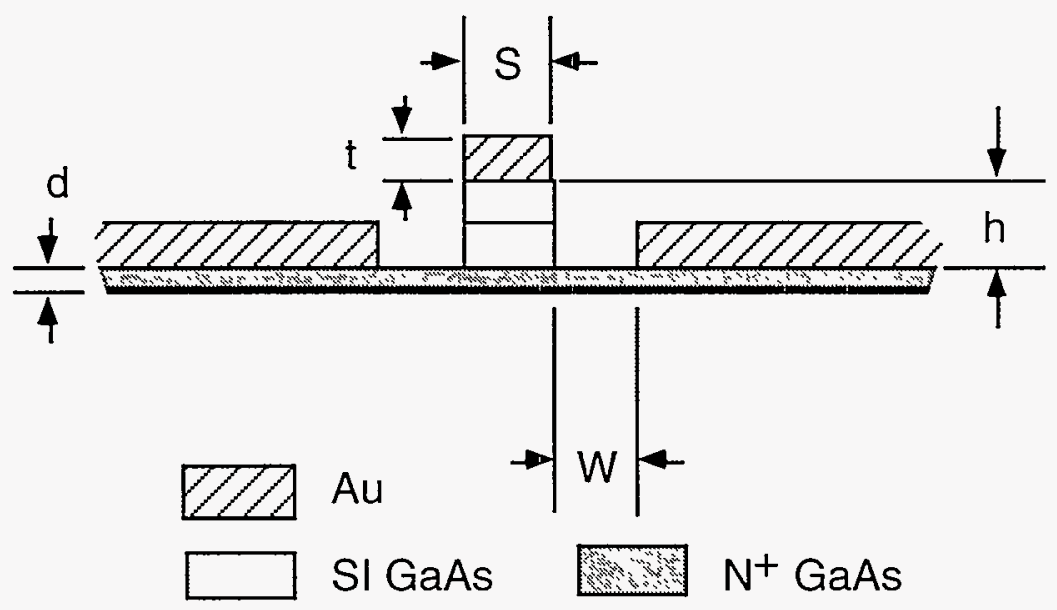

Fig. 14.Dimensioned simplified cross-section of TWPD for electrical propagation analysis.

To achieve velocity synchronism and to predict the detector's bandwidth, a model for electrical propagation along the detector is necessary. The type of transmission line formed in this detector is commonly called a slow-wave coplanar waveguide [14],[15]. Such transmission lines require "full-wave" analysis for rigorous modeling [16]. Fortunately for our purposes, quasi-TEM analysis [17],[18] satisfactorily describes these lines properties.

The equivalent circuit used for the analysis was shown in Fig. 9. The line's impedance per unit length $Z$ and admittance per unit length $Y$ must be determined. Assuming that the conductivity of the $\mathrm{n}^{+}$semiconductor is low enough to allow the skin-depth in the semiconductor to be much larger than the line's cross-sectional dimensions, the magnetic field will not "see" the buried semiconductor. Then, the inductance $L$ is identical to that of a conventional coplanar waveguide:

$$
L=\frac{\mu_{o}}{4 F}
$$

where 


$$
F=\left\{\begin{array}{l}
\ln \left(\frac{2(1+\sqrt{k})}{(1-\sqrt{k})}\right) / \pi, \text { for } 0.707 \leq k \leq 1 \\
\pi / \ln \left(\frac{2\left(1+\sqrt{k^{\prime}}\right)}{\left(1-\sqrt{k^{\prime}}\right)}\right), \text { for } 0 \leq k \leq 0.707
\end{array}\right.
$$

in which $k=S /(S+2 W)$ and $k^{\prime}=\sqrt{1-k^{2}}$ where $S$ is the width of the center strip and $W$ is the gap between the center strip and ground plane [19]. The capacitance per unit length is estimated to be that of a parallel-plate capacitor:

$$
C=\varepsilon_{o} \varepsilon_{r} \frac{S}{h}
$$

where $h$ is the thickness of the detector's depletion width and $\varepsilon_{r}$ is the relative permittivity of GaAs. Assuming that the detector will be operated at large reverse bias, the semiconductor layers in the rib will be largely depleted. Then, semiconductor losses associated with transverse current flow can be included in the model as a series resistance cause by the $n+$ layer. This resistance can be estimated as that of a simple rectangular resistor:

$$
R_{T}=\frac{1}{2}\left(\frac{W}{\sigma_{n^{+}} d}\right)
$$

where $\sigma_{n+}$ and $d$ are the conductivity and thickness of the $n^{+}$layer respectively.

The metal losses are assumed to be similar in form to those of a parallel plate waveguide:

$$
Z_{m}^{\prime}=\frac{Z_{m}}{S^{\prime}} \operatorname{coth}\left(j k_{x m} t\right)
$$

where $Z_{m}=\sqrt{\mu_{d} \varepsilon_{o} \varepsilon_{r m}}, S^{\prime}$ is an effective center strip width, and $k_{x m} \approx k_{o} \sqrt{\varepsilon_{r m}}$ in which the complex permittivity of the metal $\varepsilon_{r m}=-j \sigma_{m} / \omega \varepsilon_{o}$ where $\sigma_{m}$ is the conductivity of the metal electrodes. The effective strip width $S^{\prime}$ was found to be approximately $S$, the actual physical width of the center strip.

With these circuit elements once known, the impedance $Z$ and admittance $Y$ both per unit length are:

and

$$
Z=Z_{m}^{\prime}+j \omega L
$$

$$
Y=j \omega C+\frac{1}{R_{T}} .
$$

The complex propagation constant $\gamma$ and the complex characteristic impedance $Z_{o}$ can be found: 


$$
\gamma=\sqrt{Z Y}
$$

and

$$
Z_{o}=\sqrt{\frac{Z}{Y}} .
$$

Table 2 gives the dimensions and electrical parameters used for our first TWPD. As seen in the next section, these dimensions are amenable to the optical dimensions required. Using these dimensions and (32)-(39), Figs. 15 and 16 were generated. Fig. 15 shows a plot of the theoretical RF index and RF loss $(\mathrm{dB} / \mathrm{mm})$. The index is close to the lights $(\approx 3.5)$ and the loss is reasonably low at $\mathrm{mm}$-wave frequencies. Fig. 16 shows a plot of the theoretical characteristic impedance which is quite close to $50 \Omega$.

\begin{tabular}{|c||c|}
\hline$S$ & $3 \mu \mathrm{m}$ \\
\hline$W$ & $3 \mu \mathrm{m}$ \\
\hline$t$ & $3 \mu \mathrm{m}$ \\
\hline$h$ & $1.5 \mu \mathrm{m}$ \\
\hline$d$ & $0.5 \mu \mathrm{m}$ \\
\hline$\sigma_{a u}$ & $4.1 \times 10^{7} \mathrm{~S} / \mathrm{m}$ \\
\hline$\sigma_{N_{+}}$ & $8.3 \times 10^{4} \mathrm{~S} / \mathrm{m}$ \\
\hline$\varepsilon_{r}$ & 12 \\
\hline
\end{tabular}

Table 2. Parameters used for TWPD electrical propagation calculations.

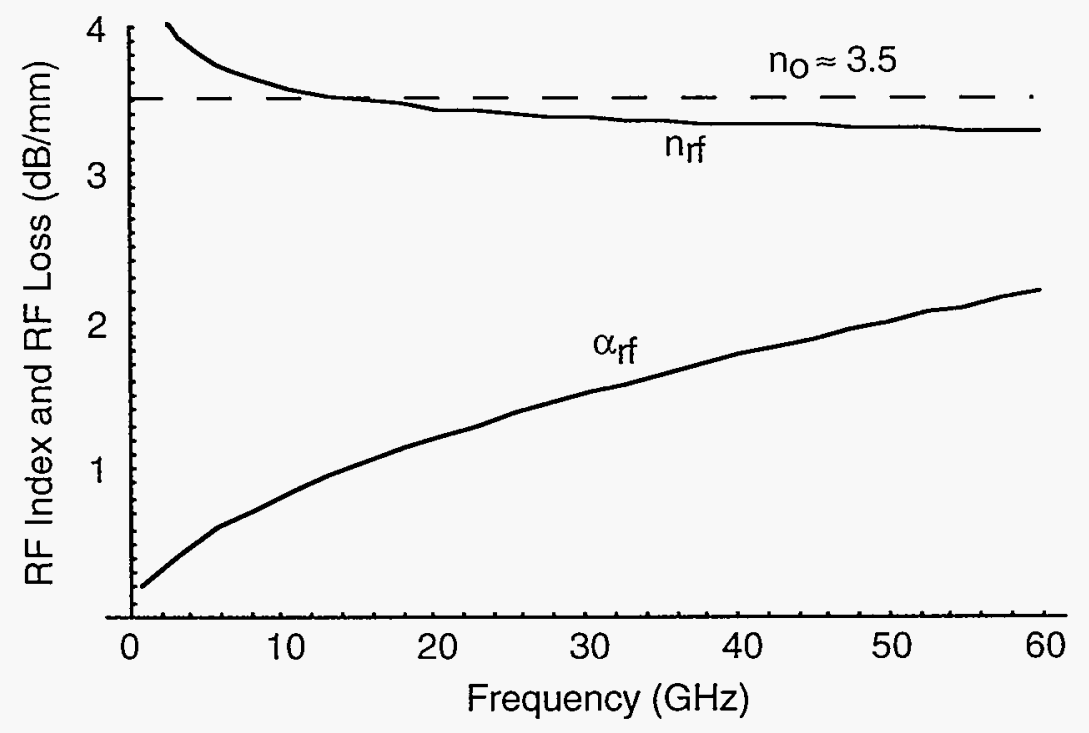

Fig. 15. Theoretical microwave RF Index and RF Loss. 


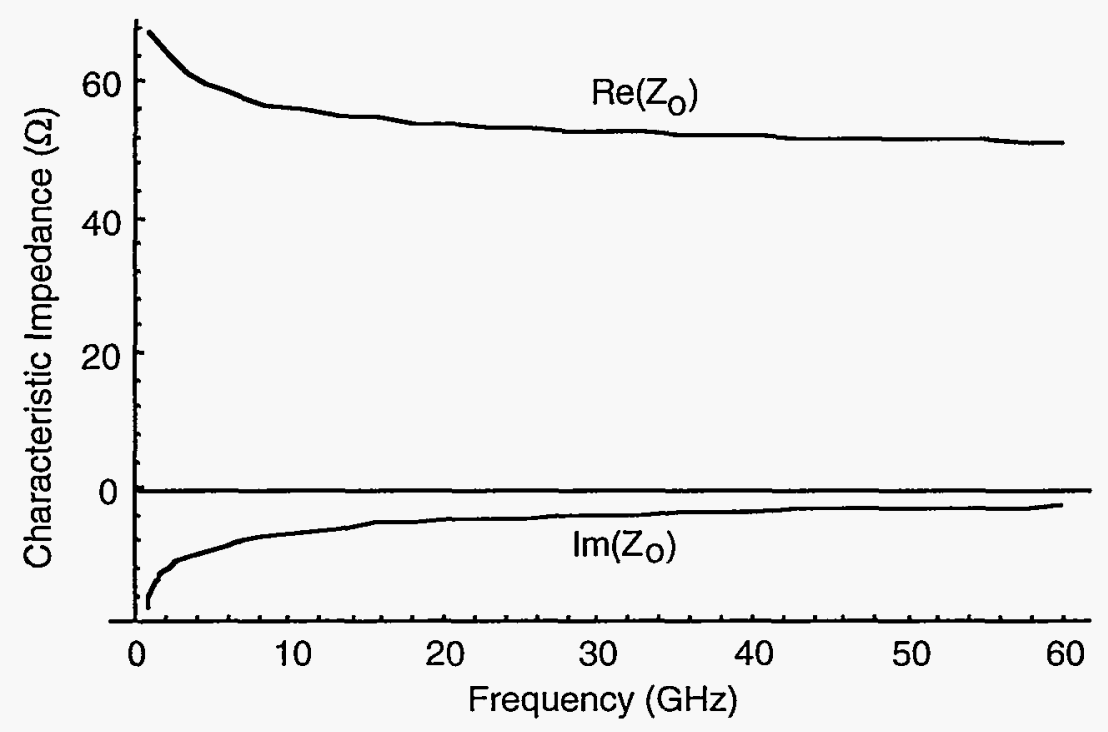

Fig. 16. Theoretical microwave characteristic impedance.

\section{Optical Design}

Our present designs are optimized for $840 \mathrm{~nm}$ and $1.06 \mu \mathrm{m}$ light. All measurement results presented in this document are using the $1.06 \mu \mathrm{m}$ design. The detector can be easily extended to other wavelengths by changing material systems. All of the discussions here as well as the fundamental operation of our device are wavelength independent.

The key operating feature of the traveling-wave detector is the ability to tailor the absorption length of the light in the waveguide so as to reduce the absorbed power density. This is done by employing a single quantum well of GaAs or InGaAs with a high absorption coefficient within an otherwise transparent waveguide. Total waveguide loss is then determined by the absorption coefficient of the quantum well multiplied by the percentage of total light in the waveguide that actually travels inside the quantum well (the overlap integral of the waveguide mode and the quantum well.) The quantum well itself must be designed so that the electron-hole excitonic resonance occurs at the desired photon energy. This determines the width and composition of the quantum well, (Table 3 ). Our final design quantum well used $30 \%$ In and a $130 \AA$ width to ensure that the absorption edge of the well is slightly lower than the desired $1.064 \mu \mathrm{m}$ operating wavelength. Room temperature absorption measurements show that the absorption coefficient of such strained quantum well is $\sim 1000 \mathrm{~cm}^{-1}$ at wavelengths slightly below the absorption edge wavelength.

As mentioned above, absorption of the waveguide mode is set by the overlap of the optical mode with the quantum well. We can adjust the overlap integral over a wide range through correct selection of the refractive index profile of the waveguide materials. We will not go into the details of the design approach other than to provide the complete detector design used in this work. 
Fig. 17 show a cross section schematic of our design for $1.06 \mu \mathrm{m}$ wavelength while Fig. 18 shows the results of a two-dimensional simulation of the optical waveguide. The simulation includes effects of absorption in the quantum well and yields an optical modal index of 3.227438 with an absorption coefficient of $30 \mathrm{~cm}^{-1}$ assuming $1000 \mathrm{~cm}^{-1}$ absorption in the quantum well region. This absorption value leads to absorption of $99 \%$ of the initial light coupled into the waveguide within $1.5 \mathrm{~mm}$ of propagation length.
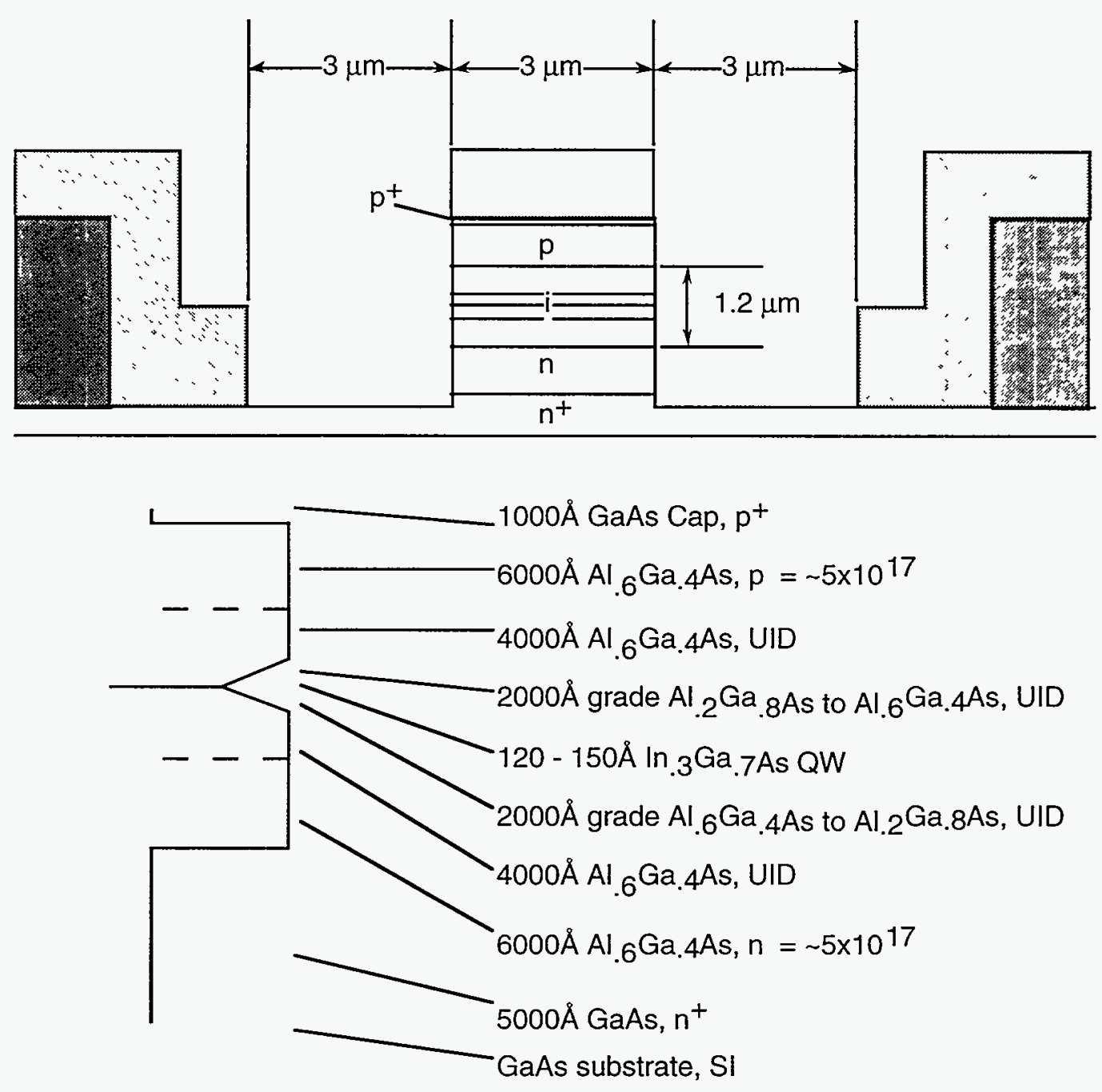

Fig. 17. Cross-section schematic of the TWPD for $1.06 \mu \mathrm{m}$ operation (top) and diagram of material composition and thicknesses through the center axis of the waveguide (bottom). 


\begin{tabular}{|l||l|l|l|}
\hline$x$ & $100 \AA$ & $\begin{array}{l}\text { Width } \\
120 \AA\end{array}$ & $150 \AA$ \\
\hline $24 \%$ & 1.0328 & & \\
\hline $25 \%$ & 1.0416 & 1.0511 & 1.0601 \\
\hline $28 \%$ & & & 1.0883 \\
\hline $30 \%$ & 1.0869 & & 1.1079 \\
\hline
\end{tabular}

Table 3. Exciton resonance of quantum well in terms of $\mu \mathrm{m}$ wavelength. Mole fraction $(x)$ is percent Indium in $\ln _{\mathrm{x}} \mathrm{Ga} a_{1-\mathrm{x}}$ As.

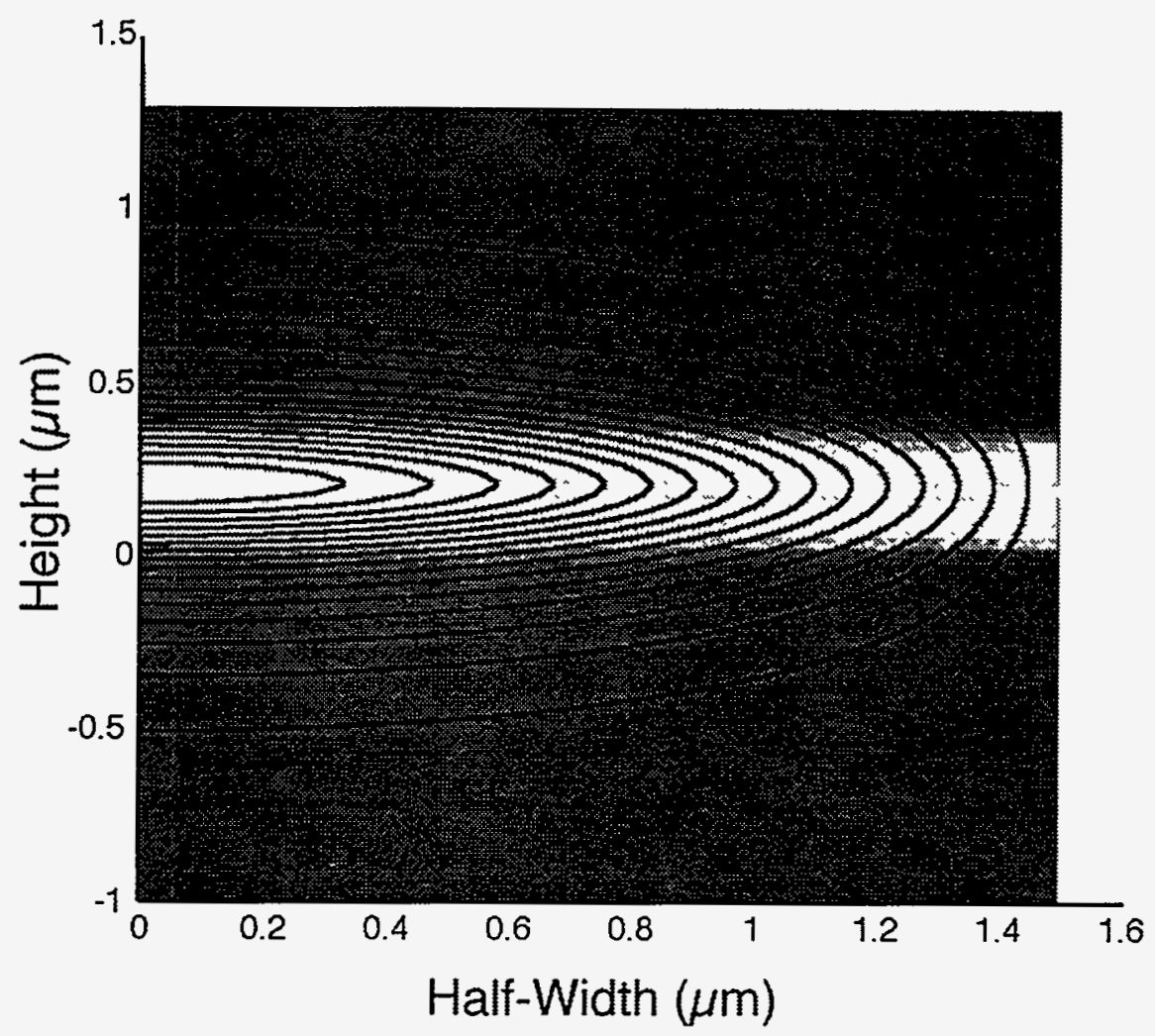

Fig. 18. Contour plot of constant electric field of the TEOO mode of our TWPD. Gray shading corresponds with Al mole fraction of the waveguide material. Darker gray is high Al content, lower refractive index. Absorption of this mode due to the quantum well is calculated to be $30 \mathrm{~cm}^{-1}$.

The device layout shown in Fig. 17 is a plot of the mask set for our device. Light enters from the right through a cleaved facet. Electrical signals are output from the contact pads on the left of the device. 


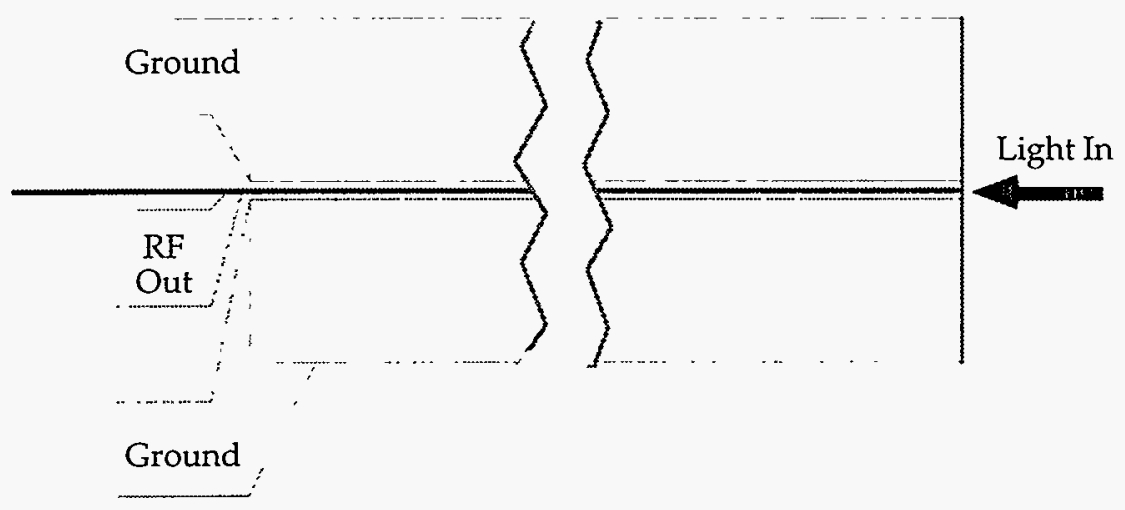

Fig. 19. Top view of Traveling-wave photodetector. Contact pads are on the left. Light enters from the right.

With the optical design selected, the RF propagation model and detector response model can be combined to predict real detector performance. The device parameters in Table 4 and Table 2 were used to generate the theoretical response plotted in Fig. 20 (single output termination). The $6 \mathrm{~dB}$ rolloff as discussed previously is still clearly present. An additional rolloff of a few dB due to RF propagation losses along the detector is also present. The ripples at high frequencies are due to the finite length $(1250 \mu \mathrm{m})$ of the detector and slight impedance differences between the termination and the detector's characteristic impedance.

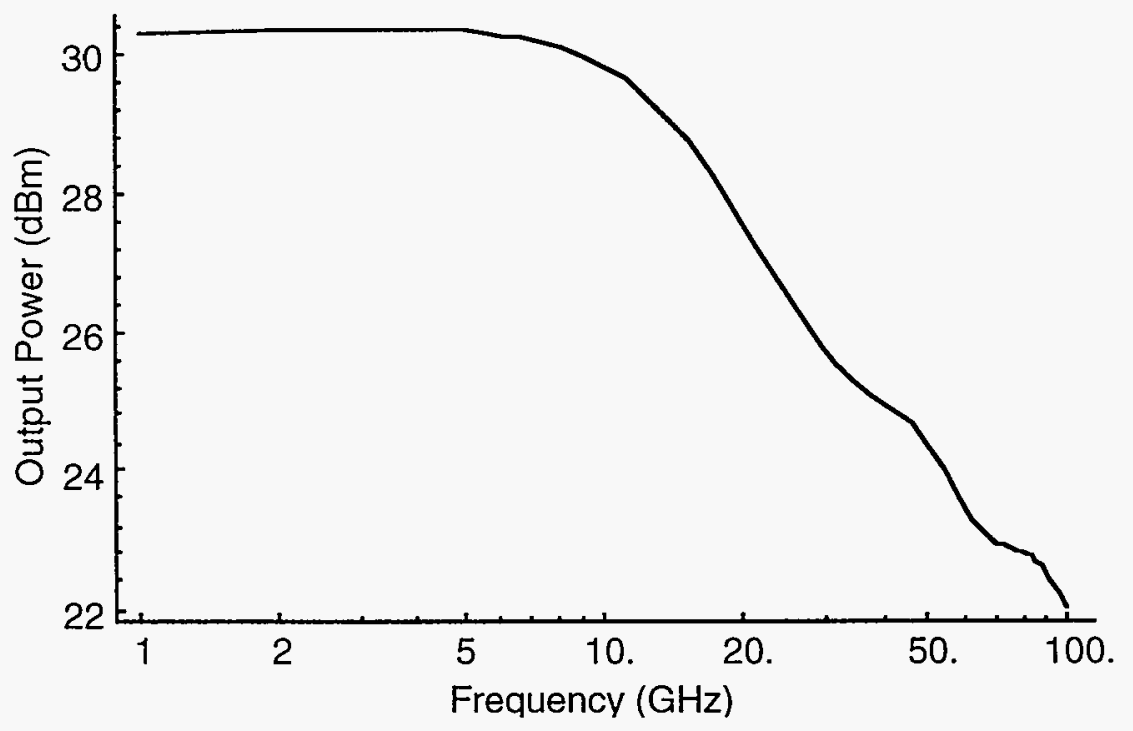

Fig. 20. Theoretical detector output power for the device parameters given in Tables 1-3. 


\begin{tabular}{|c||c|}
\hline 1 & $1250 \mu \mathrm{m}$ \\
\hline$P_{1}$ & $100 \mathrm{~mW}$ \\
\hline$\xi$ & $100 \%$ \\
\hline$\alpha_{0}$ & $1 \mathrm{~mm}^{-1}$ \\
\hline$\lambda_{0}$ & $0.840 \mu \mathrm{m}$ \\
\hline
\end{tabular}

Table 4. Parameters used for TWPD performance calculations.

\section{Detector Fabrication}

Fabrication of the TWPD proceeded as follows: The $3 \mu \mathrm{m}$ wide waveguide rib was defined by conventional contact-print photolithography using a positive-working tri-level etch mask [20],[21]. The waveguides were etched using chlorine reactive-ion-beam etching [22] (RIBE) in a load-locked configuration for equal-rate etching of GaAs and AlGaAs with smooth, vertical, sidewalls [23]. The etch was extended to the interface between the lower AlGaAs cladding layer and the lower $\mathrm{n}^{+}$-doped GaAs conductive layer. Termination of the etch just below the interface was essential to behavior of the device. If the etch were too deep the resistance between the photodiode and $n$ type ohmic contact would be too high while etching too shallow leaves wideband-gap AIGaAs between the ohmic metal and the n-type GaAs, raising the contact resistance. The required etch depth accuracy was obtained through the use of in-situ reflectometry [24]. A second mask-etch step was then used to etch away the $\mathrm{n}^{+}$layer in the regions where it was not needed.

After etching, a layer of photosensitive polyimide was spun-on to a thickness just greater than the height of the waveguide rib and openings created for the n-type ohmic contact. The top of the waveguide rib was exposed by $\mathrm{O}_{2}$ RIBE etch-back of the polyimide layer. Once the polyimide openings were created both $\mathrm{p}$ - and $\mathrm{n}$-type ohmic contacts and the microwave transmission lines were formed using lift-off metalization. The contacts were alloyed in a rapid-thermal-anneal furnace at $420^{\circ} \mathrm{C}$ for 30 seconds.

The detailed process is as follows:

1) Mask with photoresist and etch rib waveguide into the $\mathrm{n}^{+}$layer.

2) Remove previous mask, remask for mesa-isolation and etch into the semi-insulating substrate.

3) Remove mask, clean and deposit a $0.1 \mu \mathrm{m} \mathrm{SiO} 2$ layer for surface protection.

4) Planarize with photodefinable polyimide leaving openings over the ground-plane contacts.

5) Oxygen RIE etch entire polyimide surface to expose waveguide tops. 
6) Remove exposed $\mathrm{SiO}_{2}$ on waveguide tops with dilute buffered $\mathrm{HF}$ acid.

7) Deposit and pattern by lift-off a thin Ti/Au p-type ohmic metal layer over the waveguide.

8) Deposit and pattern by lift-off a thin Au/Ge/Ni/Au n-type ohmic metal layer over the $\mathrm{n}^{+}$contact area.

9) Deposit a thin Ti/Au "seed" layer over entire wafer.

10) Mask with photoresist for plate Au

11) Plate thick Au layer over both $p$ and $n$ contact metals.

12) Remove photoresist mask.

13) Etch exposed "seed" layer with Ar ion mill.

14) Lap and polish backside to desired $150 \mu \mathrm{m}$ wafer thickness.

Figs. 21-23 show electron micrographs of a completed TWPD.

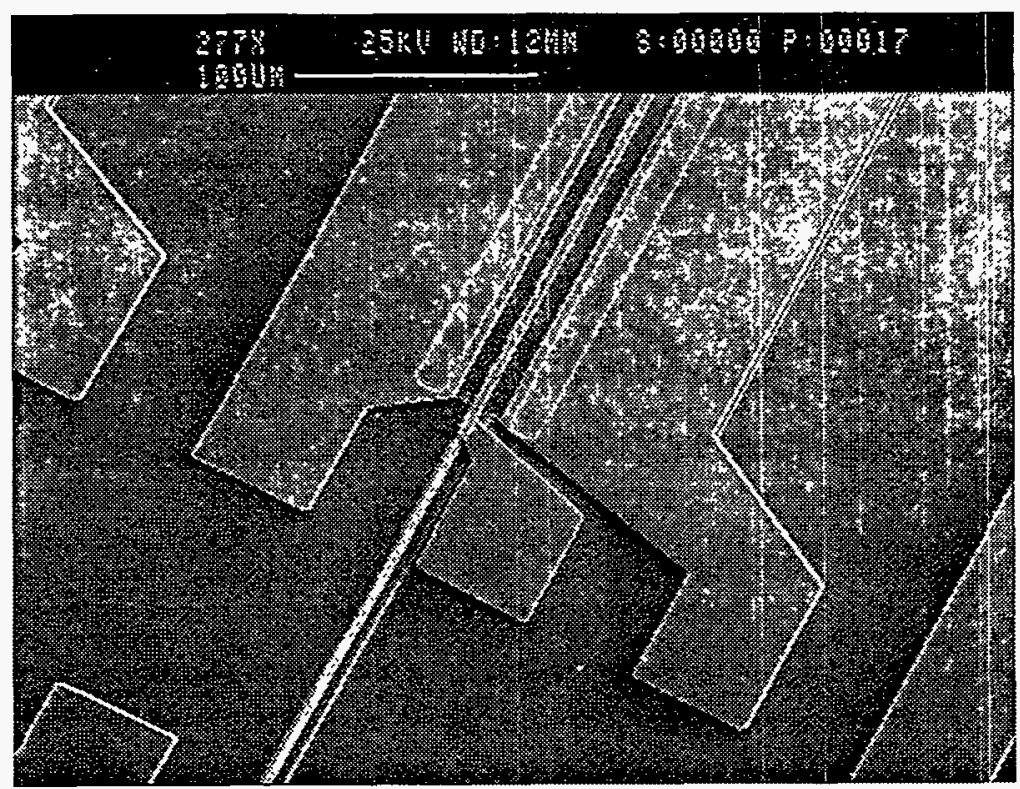

Fig. 21. SEM micrograph of top view of traveling-wave photodetector. Optical guide extends diagonally for lowerleft to upper-right at center. Center electrical contact connected to optical guide's contact is the signal. Ground lines exist to either side. 


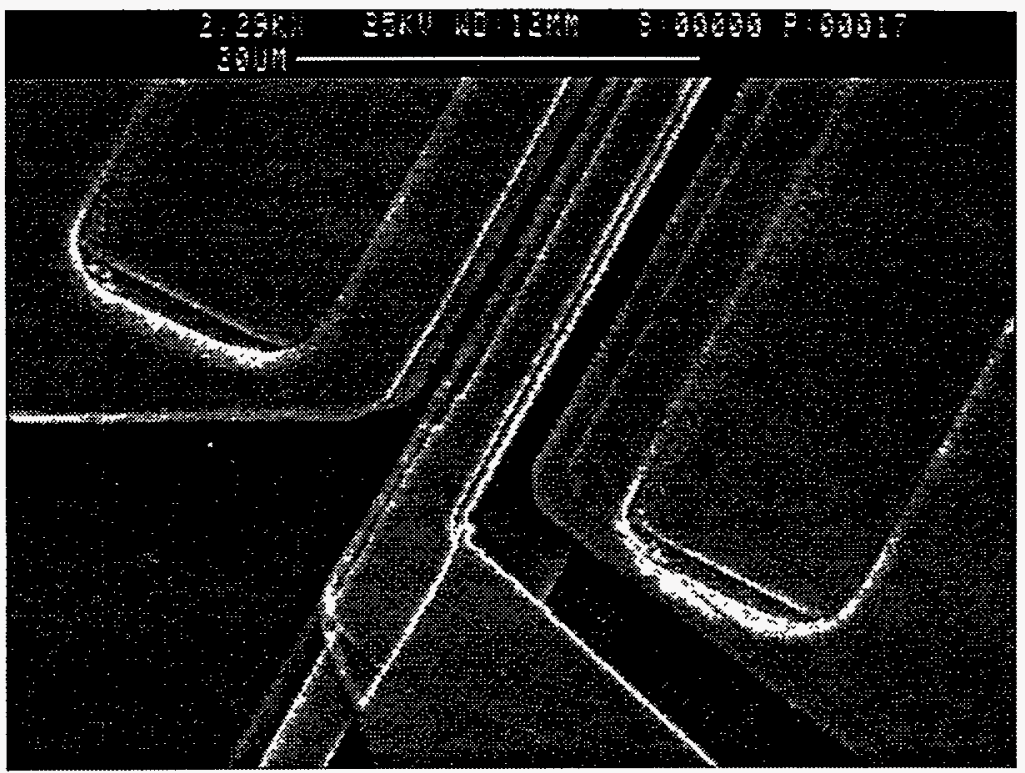

Fig. 22. SEM micrograph of electrical pad interface to center conductor of the optical guide. Ground-to-signal gaps are approximately $3 \mu \mathrm{m}$.

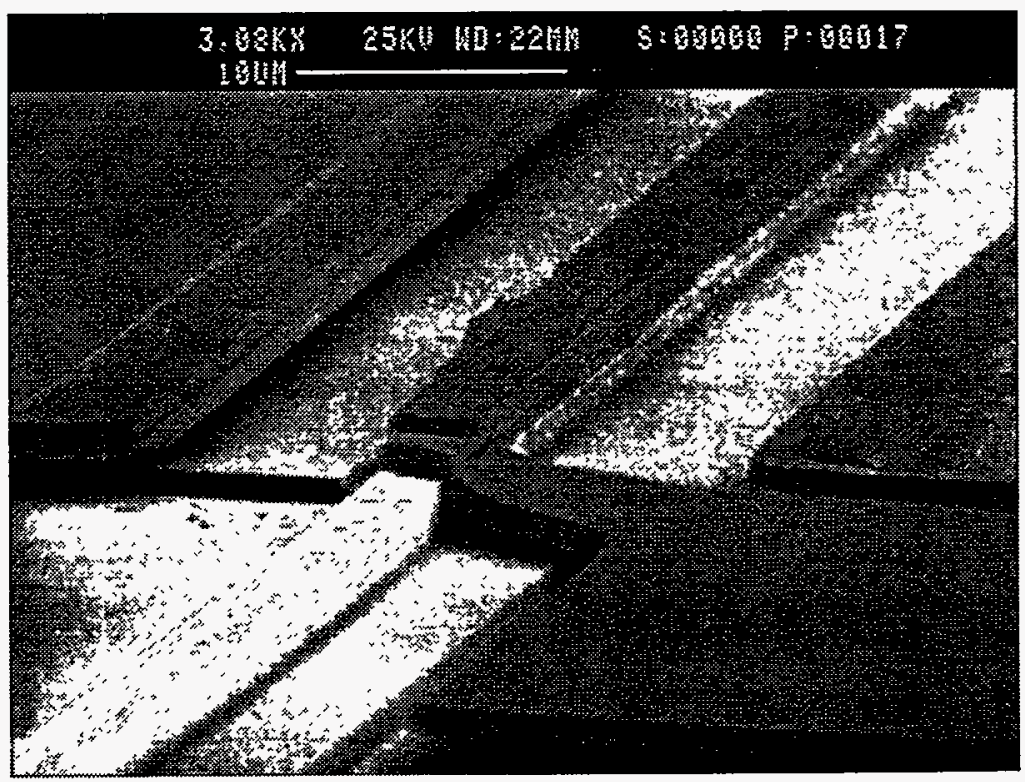

Fig. 23. SEM micrograph of "air-bridge" connection to center electrode on top of the optical guide.

\section{Detector Characterization}

Detectors were fabricated as described previously. The detectors were characterized by electrical measurements of propagation along the electrodes and by optical measurements of bandwidth. 


\section{Electrical Characterization}

Measurements of electrical propagation along the electrodes were performed by special test structures which had electrical probe contacts at both ends of the device. On-wafer microwave probes were then used to measure the electrodes two-port S-parameters. These S-parameters are then directly converted to transmission line parameters as described elsewhere [25]. Measurements on a $2.25 \mathrm{~mm}$ long test device are summarized in Figs. 24 through and 26. Fig. 24 shows a plot of the measure RF index. This can be compared to the theoretical plot given in Fig. 15. A good correspondence is seen with the measured RF index being quite close to the desired optical index (3.5). The RF index is largely bias independent due to the low doping level within the detector's junction.

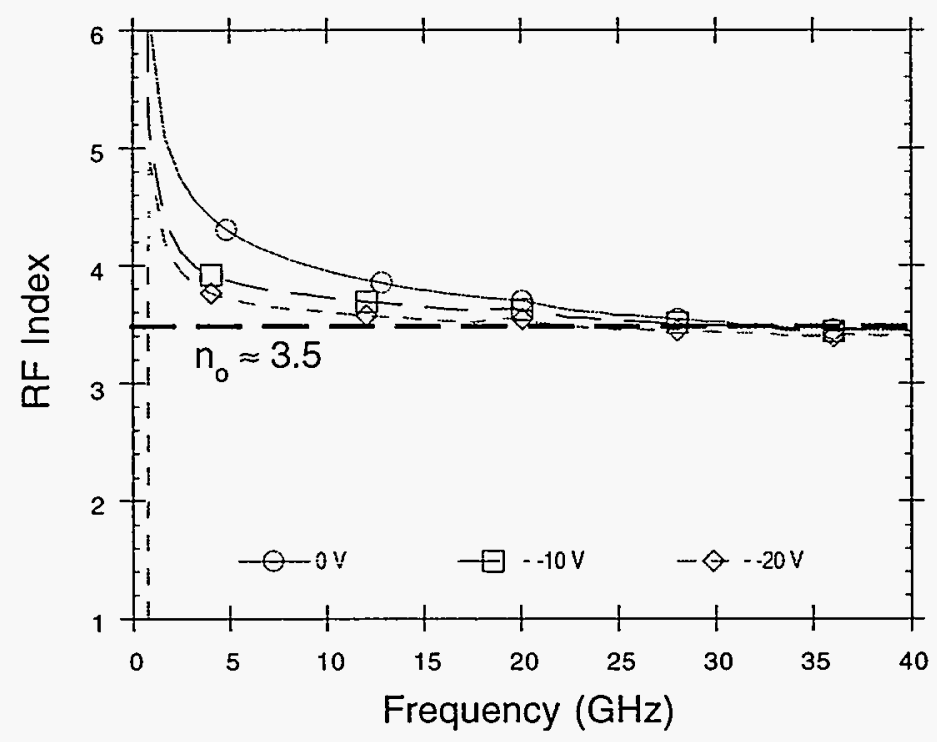

Fig. 24. Measured RF index of the TWPD. The RF index approximately matches the optical index.

Fig. 25 shows a plot of the measured characteristic impedance. This can be compared to the theoretical plot given in Fig. 16. Note that the characteristic impedance is mainly real with a approximately $50 \Omega$ value. Again, the characteristic impedance is relatively bias independent due to the low doping in the junction. The $50 \Omega$ impedance makes this device easily inserted into conventional $50 \Omega \mathrm{RF}$ circuits/systems. 


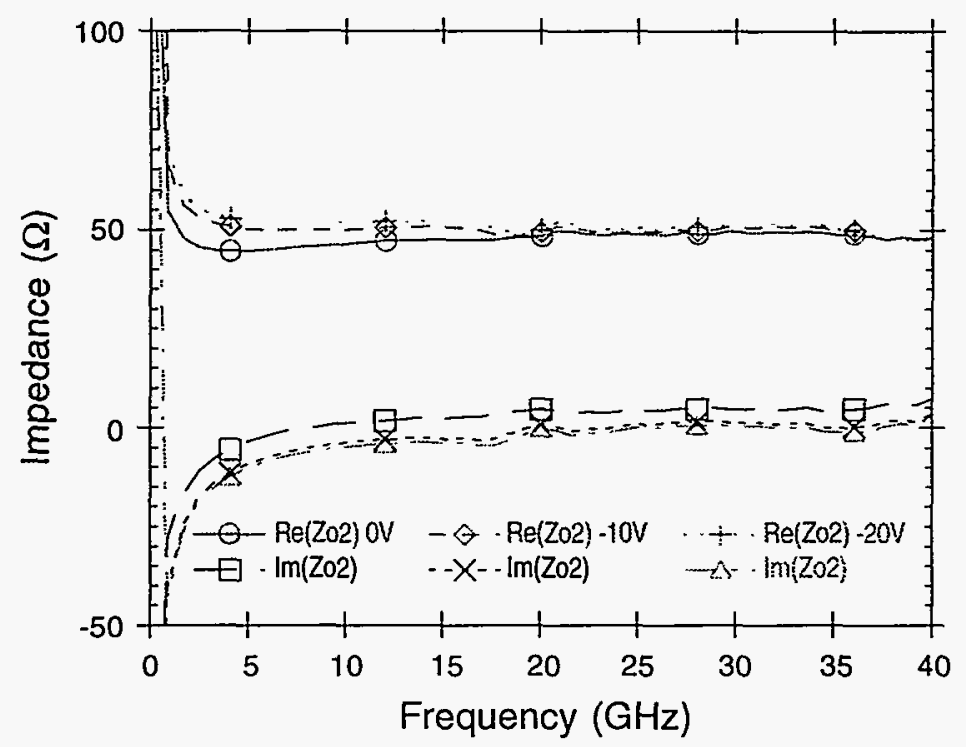

Fig. 25. Measured characteristic impedance of the TWPD. The characteristic impedance is approximately $50 \Omega$.

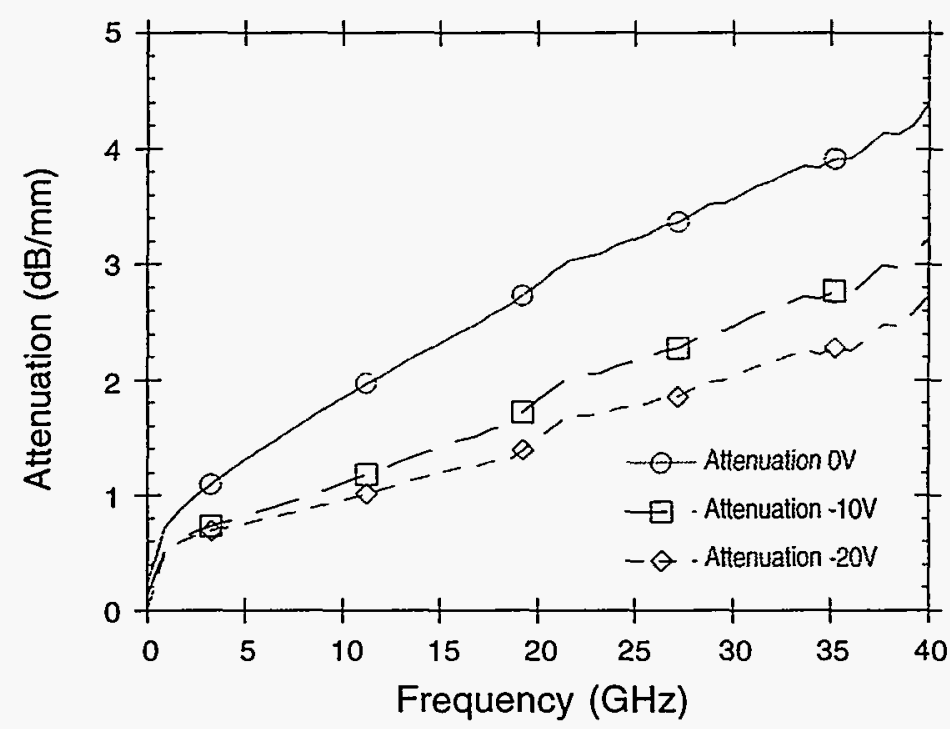

Fig. 26. Measured attenuation of the TWPD. Attenuation decreases with increased reverse bias due to depletion in the PIN junction.

Fig. 26 shows the measured attenuation of the detector. This should be compared to the theoretical plot given in Fig. 15. A good correspondence is 
seen at high biases (complete depletion) which is consistent with the assumptions made in the theory. The attenuation is adequate for this application though improvement in device performance would be seen if the attenuation could be reduced. Reduction of the attenuation is somewhat difficult as metal loss dominates the total propagation loss. Therefore, small changes to the semiconductor would have little impact on attenuation and changes to the metal electrodes are the most promising. Increasing metal thickness will to first order only lower loss at low frequencies due to the skin effect. On the other hand, increased center strip width would lower losses and appears to be the most viable approach.

\section{Optical Bandwidth Measurements}

A two laser optical heterodyne system was constructed for electrical bandwidth measurements (see Fig. 5). Nd:YAG lasers at $1.06 \mu \mathrm{m}$ were used for the light sources because of their extremely narrow line widths $(<10 \mathrm{KHz})$. The lasers ${ }^{\prime}$ wavelengths were tuned by varying the temperature of the lasers' cavities. A mode-hop-free rf-beat-frequency range of about $60 \mathrm{GHz}$ is possible with commercial Nd:YAG lasers from Lightwave Technologies [26]. Actual measurements were performed by fixing the temperature on one laser and then adjusting the temperature of the other, waiting for thermal equilibrium, and then measuring the resulting frequency and power level from the detector with an if spectrum analyzer.

Fig. 27 shows a measured response from a $1.25 \mathrm{~mm}$ TWPD. This detector was operated with the input end electrically open and the output side terminated with the $50 \Omega$ load (the spectrum analyzer). Bandwidth is seen to be approximately only $4.8 \mathrm{GHz}$. This bandwidth is significantly less than that expected based on the theory derived in this report $(\approx 20 \mathrm{GHz})$. The bandwidth does not appear to be limited by the propagation losses along the electrodes, as indicated by the measurements presented in the previous section. The present bandwidth limitation is believed to be due to carrier transit time across the detector's depletion regions.

Transit time effects have been neglected in the analysis presented to this point. This approximation is justified provided that the transit time is much less than the period of the operating frequency. This requirement can be achieved by making the detector "thin" in the direction of carrier transport. The transit-time cut-off frequency $f_{t}$ for a PIN photodetector is given by [27]:

$$
f_{t}=0.533 \mathrm{v} / \mathrm{W}
$$

in which $v$ is the average of the peak carrier velocities for electrons and holes in the semiconductor, and $W$ is the width of the depletion region. For GaAs this velocity is about $1.86 \times 10^{7} \mathrm{~cm} / \mathrm{s}$ [27]. For the material structure used for our present TWPD, carrier transport is through graded AlGaAs which would be expected to have a lower carrier velocity. The depletion width $(W)$ is 
approximately $2.5 \mu \mathrm{m}$ thick. This with the velocity of GaAs and using (39), gives a cutoff frequency of approximately $40 \mathrm{GHz}$. This is much greater than the observed cuttoff frequency of $4.8 \mathrm{GHz}$, but the velocity in AlGaAs my be many times less than that in GaAs. Additionally, after including the $6 \mathrm{~dB}$ intrinsic response rolloff, the electrical losses along the detector, and the possible measurement errors, the response in Fig. 25 is not all that unreasonable. Nevertheless, future designs should have a thinner depletion thickness to reduce carrier transit times for operation into the millimeter wave frequency regime.

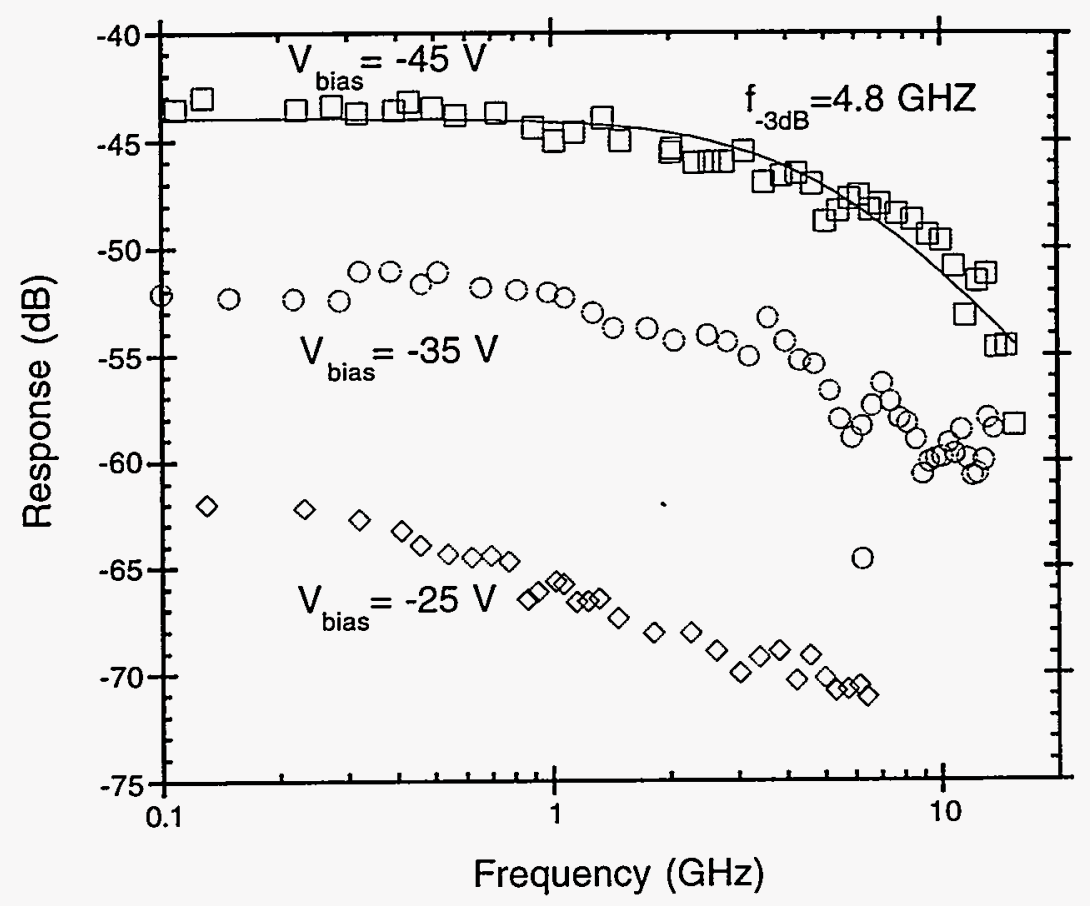

Fig. 27. Response measurement of the TWPD. Bandwidth is approximately $4.8 \mathrm{GHz}$.

\section{System Example}

This section presents a hypothetical RF generation system which could be fabricated with our traveling-wave photodetector. Laser light power is produced by two commercial CW single-line lasers from Spectra-Diode Labs [27]. The solid-state diode lasers produce $100 \mathrm{~mW}$ each at $838 \mathrm{~nm}$ with an efficiency of about $30 \%$. The lasers' wavelengths are assumed to be temperature tuned to produce the necessary RF beat frequency.

Fig. 28 shows a dual laser RF generator system employing a travelingwave photodetector. This type of system was discussed extensively earlier in this report. Such a system would be able to operate up to the RF bandwidth of 
the photodetector. This is expected to be ultimately well into the millimeter wave frequency range $(>60 \mathrm{GHz})$.

From (7), the expected RF output power would be $456 \mathrm{~mW}$. Recall that the RF output power is quadratically dependent on the optical power. Therefore, if the lasers' power was doubled (a realistic near term expectation) the output power would increase by a factor of 4 . The ideal bias point for the traveling-wave photodetector can be determined by (12); giving $6.75 \mathrm{Vdc}$. The detector's power dissipation will be at most $656 \mathrm{~mW}$ as found from (15). With this value, the maximum system efficiency is about $34 \%$. This efficiency is undoubtedly an optimistic estimate as all system losses have been neglected. In an actual system, there would be optical as well as RF losses which both reduce the output power and therefore the efficiency.

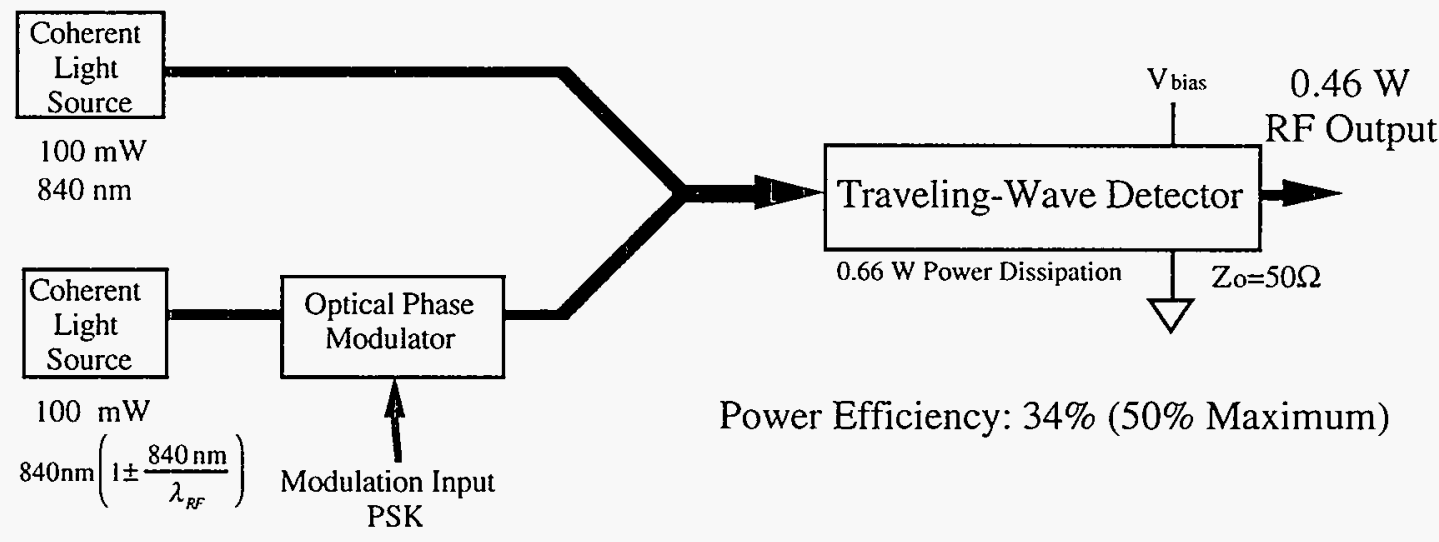

Fig. 28. Example: Dual Laser System.

The system in Fig. 28 shows optical phase modulation of one of the lasers' outputs. This would provide a simple means of modulating the phase of the RF output. Any relative optical phase shift introduced into either laser output will map directly into an RF phase shift at the output. Therefore, Phase Shift Keying (PSK) of the RF output is achieved by varying the bias on the optical phase shifter.

\section{Conclusions}

RF power generation by optical heterodyning is a viable technique provided adequate laser power and suitable photodetectors are available. Suitable laser power already exists for significant RF power levels to be produced. Additionally, laser power will undoubtedly continue to increase with the maturity of laser diode technology. No photodetector exists today which can withstand the power levels required. The traveling wave photodetector would be able to handle these large power levels while maintaining a large electrical bandwidth. The traveling-wave photodetector is the critical component for the successful generation of RF power by optical means. 


\section{References}

[1] M. Maeda, T. Hirata, M. Suehiro, M. Hihara, A. Yamaguchi, and H. Hosomatsu, "Photonic Integrated Circuit Combing Two GaAs Distributed Bragg Reflector Laser Diodes for Generation of the Beat Signal," Jpn. J. Appl. Phys., vol. 31, pp. L183-L185, 15 Feb. 1992.

[2] G. J. Simonis, and K. G. Purchase, "Optical Generation, Distribution, and Control of Microwaves Using Laser Heterodyne," IEEE Trans. Microwave Theory Tech., vol. 38, pp. 667-669 (1990).

[3] J. J. O'Reilly, P. M. Lane, R. Heidemann, and R. Hifstetter, "Optical Generation of Very Narrow Linewidth Millimeter Wave Signals," Electron. Lett., vol. 28, pp. 2309-2311, 3 Dec. 1992.

[4] Anritsu Model MG9505A/MG9603A Heterodyne Optical Sweeper, provides $0.5 \mathrm{~mW}$ optical output and 10's of $\mu$ W RF output.

[5] E. R. Brown, K. A. Mclntosh, F. W. Smith, K. B. Nichols, M. J. Manfra, and C. L. Dennis, "Optical-heterodyne generation in low-temperature-grown FaAs and InGaAs", paper 2145-28 presented at OE/LASE '94, Los Angles, CA, January 22-29, 1994.

[6] Kul B. Bhasin, and A. S. Daryoush, From Short Cource Notes, SC40, Lightwave Technology for Microwave Systems: Principlies and Applications, at SPIE's OE LASE'90, Los Angeles, CA, January 15,1990.

[7] Anritsu Model MG9505A/MG9603A Heterodyne Optical Sweeper, provides $0.5 \mathrm{~mW}$ optical output and 10's of $\mu$ W RF output.

[8] C. S. Tsai, Chapter 5 "Optical Modulation: Acousto-Optical Devices", Handbook of Microwave and Optical Components, edited by K. Chang, pp. 199-246, John Wiley \& Sons, 1991.

[9] B. J. V. Zeghbroeck, W. Patrick, J. M. Halbout, and P. Vettiger, "105-GHz Bandwdith Metal-Semiconductor-Metal Photodiode," IEEE Electron Devices Letters, Vol. EDL-9, No. 10 , October 1988, pp. 527-529.

[10] V. M. Hietala, and K. S. Champlin, "Measurement of the Microwave Properties of Micron-Sized Coplanar Transmission Lines", Journal of Electromagnetic Waves and Applications, Vol. 5, No. 4/5, 439-452, 1991.

[11] V. M. Hietala, and G. A. Vawter, "A Large-Bandwidth High-Quantum-Efficiency TravelingWave Photodetector Base on a Slow-Wave Coplanar Transmission Line", presented at Progress In Electromagnetic Research Symposium, Boston, MA, July 1-5, 1991.

[12] K. S. Giboney, M. J. W. Rodwell, and J. E. Bowers, "Traveling-Wave Photodetectors," IEEE Photon. Tech. Lett., vol. 4, pp. 1363-1365, Dec. 1992.

[13] S. Ramo, J. R. Whinnery and T. Van Duzer, Fields and Waves in Communication Electronics, p. 25, John Wiley \& Sons, New York 1965.

[14] H. Hasegawa, and H. Okizaki, "M.I.S. and Schottky slow-wave coplanar striplines on GaAs substrates", Electronic Letters, Vol. 13, No. 22, 663-664, Oct. 1977. 
[15] T. Fukuoka, and T. Itoh, "Analysis of slow-wave phenomena in coplanar waveguide on a semiconductor substrate", Electronic Letters, Vol. 18, No. 14, 589-590, July 1982.

[16] Y. Fukuoka, Y. Shin, and T. Itoh, "Analysis of slow-wave coplanar waveguide for monolithic integrated circuits", IEEE Microwave Theory and Tech., Vol. MTT-31, 567573 , July 1983.

[17] C. Sequinot, P. Kennis, and P. Pribetich, "Desktop computer appraisal of potential slowwave propagation characteristics for Schottky coplanar lines", Electronic Letters, Vol. 19, No. 25 1065-1067, Dec. 1983.

[18] Y. R. Kwon, V. M. Hietala, and K. S. Champlin, "Quasi-TEM analysis of slow-wave mode propagation on coplanar microstructure MIS transmission lines", IEEE Microwave Theory Tech., Vol. MTT-35, June 1987.

[19] C. Gupta, R. Garg, and I. J. Bahl, Microstrip Lines and Slotlines, pp. 260-262, Artech House, Norwood, MA, 1979.

[20] K. Asakawa and S. Sugata, "GaAs AlGaAs anisotropic fine pattern etching using a new reactive ion beam etching system", J. Vac. Sci. Tech B3(1), pg. 402 (1985).

[21] G. A. Vawter, L. A. Coldren, J. L. Merz and E. L. Hu, "Nonselective etching of GaAs/AIGaAs double heterostructure laser facets by $\mathrm{Cl}_{2}$ reactive ion etching in a load-locked system", Appl. Phys. Lett. 51(10), pp. 719-721 (1987).

[22] K. Asakawa and S. Sugata, "GaAs and GaAlAs equi-rate etching using a new reactive ion beam etching system", J.J. Appl. Phys. 22(10), pg. L653 (1983).

[23] G. A. Vawter, J. F. Klem, G. R. Hadley and S. H. Kravitz, "Highly Accurate etching of ridge-waveguide directional couplers using in-situ reflectance monitoring and periodic multilayers", Appl. Phys. Lett. 62(1), pp. 1-3 (1993).

[24] G. A. Vawter, J. F. Klem and R. A. Leibenguth, "Improved Epitaxial Layer Design for Real-Time Monitoring of Dry-Etching in III-V Compound Heterostructures with Depth Accuracy of $\pm 8 \mathrm{~nm} "$, J. Vac. Sci. Technol. A 12(4), (to be published) (1994).

[25] V. M. Hietala, and K. S. Champlin, "Measurement of the Microwave Properties of Micron-Sized Coplanar Transmission Lines", Journal of Electromagnetic Waves and Applications, Vol. 5, No. 4/5, 439-452, 1991.

[26] Lightwave Electronics, 1161 San Antonio Rd., Mountain View, CA 94043.

[27] Model SDL-5400, Spectra-Diode Labs,80 Rose Orchard Way, San Jose, CA 951341356. 


\section{Appendix, LDRD Summary}

Refereed publications resulting for the work: None

All other reports and publications resulting from the work: 1

V. M. Hietala, and G. A. Vawter, "A Large-Bandwidth High-Quantum-Efficiency Traveling-Wave Photodetector Base on a Slow-Wave Coplanar Transmission Line", presented at Progress In Electromagnetic Research Symposium, Boston, MA, July 1-5, 1991.

Number of patent disclosures: 1

"Traveling-Wave Photodetector", SD-4942, S-73,025

Number of patent applications: 1

Serial No. 898,999

Number of patents: 1

U. S. Patent No. 5,270,532, "Travelling Wave Photodetector" issued December 14,1993

Number of Copyrights: None

Number of students: None

Number of post docs: None

Number of permanent technical or scientific staff: None

Number of awards (and their names): None

Number of new non-LDRD funded projects and their amounts: None 


\section{Distribution:}

$1 M S 03201010$ LDRD Office

1 MS1079 1300 P.S. Peercy

$1 \quad$ MS1078 $1303 \quad$ D. R. Myers

1 MS0351 $1315 \quad$ J. J. Wiczer

$1 \quad$ MS1078 $1321 \quad$ H. T. Weaver

1 MS0603 1322 T. E. Zipperian

1 MS0603 1311 J.Y.Tsao

1 MS0603 1312 A. Owyoung

15 MS0603 1322 G. A. Vawter

15 MS0603 1322 V. M. Hietala

2 MS0603 $1311 \quad$ T. M. Brennan

2 MS0603 1311 B. E. Hammons

2 MS0874 1342 W. J. Meyer

1 MS0161 11500 Patent Office

1 MS9018 8523-2 Central Technical Files

5 MS0899 7141 Technical Library

1 MS0619 7151 Technical Publications

10 MS0100 7613-2 Document Processing for DOE/OSTI 